\title{
La “Description de l'Égypte" e le sue fonti
}

\section{Barbara Pellegrinelli}

\section{(2) OpenEdition}

\section{Journals}

\section{Edizione digitale}

URL: http://journals.openedition.org/studifrancesi/9731

DOI: 10.4000/studifrancesi.9731

ISSN: 2421-5856

\section{Editore}

Rosenberg \& Sellier

\section{Edizione cartacea}

Data di pubblicazione: 1 octobre 2007

Paginazione: 306-333

ISSN: 0039-2944

\section{Notizia bibliografica digitale}

Barbara Pellegrinelli, «La "Description de l'Égypte" e le sue fonti», Studi Francesi [Online], 152 (LI | II) | 2007, online dal 30 novembre 2015, consultato il 07 janvier 2021. URL: http://

journals.openedition.org/studifrancesi/9731 ; DOI: https://doi.org/10.4000/studifrancesi.9731

\section{(c) $(1)(9)$}

Studi Francesi è distribuita con Licenza Creative Commons Attribuzione - Non commerciale - Non opere derivate 4.0 Internazionale. 


\section{La 'Description de l'Égypte' e le sue fonti}

Poco più di duecento anni fa l'esercito francese si accingeva ad abbandonare mestamente la terra dei Faraoni, dopo una permanenza durata tre anni e due mesi, dal 2 luglio 1798 al 2 settembre 1801. Quella che è universalmente conosciuta come la spedizione d'Egitto ${ }^{1}$ ha inaugurato nell'Ottocento una nuova epoca di interesse e curiosità, di viaggi con finalità scientifiche ed archeologiche, di missioni e spedizioni ufficialmente organizzate e patrocinate dai governi europei, anelanti a riportare in vita i fasti e gli splendori del passato, e a favorire il progresso delle conoscenze tecnico-scientiche. Essa ha inoltre generato una notevole eco culturale nell'ambito dei movimenti letterari ed artistici, del gusto estetico e dell'immaginario collettivo europei. Tutto ciò ha poi trovato concreta realizzazione in numerose pubblicazioni di indiscusso interesse, e nella fondazione dell'Egittologia come scienza autonoma a partire dalle riflessioni di Champollion circa il ritrovamento della Stele di Rosetta e dalla decodificazione della scrittura geroglifica. Ma il segno più indelebile che la spedizione ci ha lasciato è indiscutibilmente rappresentato da uno dei più imponenti monumenti culturali e scientifici dell'editoria francese: la Description de l'Égypte, ou recueil des observations et recherches qui ont été faites en Égypte pendant l'expédition de l'Armée Française ${ }^{2}$. Questa grande realizzazione è stata definita da Hubert Bari ${ }^{3}$ «généreuse par sa dimension quasi-pharaonique, novatrice dans son didactisme, rigoureuse en sa démarche». Le oltre settemila pagine di memorie, osservazioni e descrizioni che costituiscono l'opera sono riunite in 9 volumi in-folio di testo, più un ulteriore volume contenente la Préface Historique, un Avertissement dell'editore ed una Explication des planches. I 9 volumi di testo sono suddivisi in tre parti: Antiquités, État Moderne e Histoire Naturelle, comprendenti rispettivamente 4, 3 e 2 volumi. All'archeologia, come si può notare, è dedicata la parte principale dell'opera. I 13 vo-

(1) Per l'inquadramento storico-politico e militare della Campagna d'Egitto si vedano soprattutto i seguenti volumi: CHARLES NORRY, Relation de l'Expédition d'Égypte, suivie de la description de plusieurs monumens de cette contrée, Paris, Pougens, an VII (1799), C. DE LA JONQUIÈRE, L'Expédition d'Égypte, 1798-1801, 1899-1907, 5 voll., H. CHARLes-Lavauzelle, Prosper JolloIs, Journal d'un ingénieur attaché à l'Expédition d'Égypte (1798-1802), Paris, Leroux, 1904, FrançoIS Charles-Roux, Les origines de l'Expédition d'Égypte, Paris, 1910, Alberto Lumbroso, Napoleone e il Mediterraneo. Vent'anni di guerra oceanica tra la Gran Bretagna e la Francia, Genova, De Fornari \& C., 1934, pp. 15 20, Christopher Herold, Bonaparte in Egypt, New York, Harper, 1962, BENOIST MÉCHIN, Bonaparte en Égypte ou le rêve inassouvi, 1797-1801, Paris, Perrin, 1978, Jean Trainié, J. C. Carmignani, Bonapar- te: la Campagne d'Égypte. Préface de Jean Toulard, Paris, Pygmalion, 1988, Henry Laurens, Charles Gillispie, L'Expédition d'Égypte, Paris, Colin, 1989, Yves LAISSUS (a cura di), Il y a 200 ans, les savants en Égypte, Paris, Nathan, 1998, Patrice BrET, L'Égypte au temps de l'expédition de Bonaparte: 1798-1801, Paris, Hachette, 1998.

(2) Description de l'Égypte, ou recueil des observations et recherches qui ont été faites en Égypte pendant l'expédition de l'Armée Française, publié par les ordres de S. M. l'empereur Napoléon le Grand, Paris, Imprimerie Impériale 1809-1813, Imprimerie Royale, 1818-1828, 10 voll. di testo in-folio, 12 voll. di tavole e 1 atlante geografico in-folio grande. Quest'opera d'ora in avanti verrà citata come $D e$ scription de l'Égypte. Ne esiste anche una seconda edizione, Paris, Panckoucke, 1820-1830, 26 voll. di testo in- $8^{\circ}, 12$ voll. di tavole in-folio. 
lumi di tavole che corredano l'opera, contenenti più di 900 incisioni, rispecchiano la stessa partizione del testo per un totale di 5 volumi di Antiquités, 2 di État Moderne e 3 di Histoire Naturelle in formato in-folio grande, più 2 ulteriori volumi di tavole ed 1 atlante geografico in formato ancor maggiore. La prima edizione dell'opera, apparsa a Parigi dal 1809 al 1828, fu tirata in mille esemplari ${ }^{4}$ e richiese il paziente lavoro di una squadra di circa 80 artisti e numerosi studiosi coordinati da otto tra i più illustri membri dell'Institut d'Égypte e della Commission des Sciences et des Arts. Voluta e concepita dal generale Kléber, il quale si operò per riunire tutta la preziosa documentazione raccolta durante la permanenza del contingente francese in Egitto in un'unica grande pubblicazione, l'opera riscosse un tale successo da richiedere addirittura una seconda edizione, meno pretenziosa e costosa rispetto alla prima, che non a caso era stata definita "impériale". Curata dall'editore e stampatore parigino Charles-Louis Panckoucke, questa seconda edizione apparve tra il 1821 e il 1830 in seguito a un'ordinanza di re Luigi XVIII del 23 giugno 1820. Tirata anch'essa in mille esemplari, questa volta la Description fu suddivisa in 26 volumi in-ottavo di testo e in 12 volumi di tavole 6 . Lo stesso Panckoucke, nella premessa alla seconda edizione, affermerà che, benché l'Egitto fosse stato oggetto di numerose descrizioni e di un gran numero di opere, non si possedeva ancora una conoscenza esatta e completa di quel paese, sorgente a cui i Greci avevano attinto i principi delle leggi, delle arti e delle scienze. Era dunque toccato alla Francia, dopo aver compiuto tutti gli sforzi per la conquista militare di questo paese, impiegare le proprie abilità artistiche e scientifiche per la sua descrizione. Un gran numero di disegnatori, di pittori, di abili tipografi, di meccanici e circa quattrocento incisori si dedicarono così, con ammirevole perseveranza, alla realizzazione di questo monumento che, sempre secondo Panckoucke, riunisce i ricordi dell'antico Egitto e la gloria della Francia moderna?

Benché oggettivamente conclusasi con il totale fallimento degli obiettivi militari e politici della Francia, la spedizione d'Egitto e il "sogno orientale" di Napoleone

(3) Hubert Bari, La Description de l'Égypte, in AA. Vv., Mémoires d'Égypte: hommage de l'Europe à Champollion, Strasbourg, La Nuée Bleue, 1990, p. 45 .

(4) Data la relativamente limitata tiratura in cui apparve, solo pochi in Europa poterono possedere una copia della Description de l'Égypte. Forse anche per questo motivo, il poterla esporre nella propria collezione costituì motivo di vanto e segno distintivo di prestigio sociale, come dimostrato dai numerosi esemplari di cui si dotarono notabili e biblioteche pubbliche.

(5) Immediatamente dopo il ritorno dell'Armata d'Oriente, il Governo ordinò con un decreto datato 6 febbraio 1802 che le memorie, le carte, i disegni e tutte le osservazioni relative alle scienze e alle arti, fatte nel corso della spedizione fossero pubblicate a vantaggio della collettività. Il lavoro di revisione e controllo di questo materiale fu affidato ad una commissione di otto persone, designata dal Ministro degli Interni e costituita da MM. Berthollet, Conté, Constaz, Desgenettes, Fourier, Girard, Lancret e Monge. Conté e Lancret furono poi sostituiti da Jomard e Jollois, mentre Delile e Devilliers furono annessi alla Commissione a partire dall'anno 1810. Il compito di commissario speciale incaricato di sorvegliare l'esecuzione, l'uniformità e l'ordine di tutte le parti dell'opera, di scegliere gli incisori, di presentare alla Commissione un resoconto delle spese e dell'avanzamento dei lavori, infine di dirigere $\mathrm{i}$ lavori di incisione e stampa fu affidato a M. Conté, che morì nel 1805. Lo sostituì, per incarico del Ministro, Michel-Ange Lancret, ingegnere civile, distintosi per le rare conoscenze di alta geometria e filosofia naturale. Egli morì nel 1807, sostituito da Jomard, già ingegnere del catasto e del deposito di guerra. Infine, la funzione di segretario incaricato della corrispondenza generale, di redigere le deliberazioni, di sorvegliare la stampa delle memorie e di concorrere insieme al commissario alla composizione e alla correzione delle tavole, fu assunta prima da Lancret, poi da Jomard, infine da Jollois, ingegnere civile. Cfr. Description de l'Égypte, Préface et explication des planches, 1809 , Avertissement, p. 1.

(6) Paul-Marie Grinevald, La Description de l'Égypte, un monument éditorial, in PATRICE BRET (a cura di), L'expédition d'Égypte: une entreprise des lumières, 1798-1801, Actes du colloque international organisé par l'Académie des Inscriptions et Belles Lettres..., Paris, Tec \& Doc, 1999, pp. 297-305.

(7) Franco Serino, Description de l'Égypte. La spedizione di Napoleone alla scoperta dell'antico Egitto, Vercelli, Ed. White Star, p. 16. 
sono ancora oggi in grado di evocare un immaginario glorioso e leggendario di prestigiose vittorie. Tuttavia, quell'aura di trionfo che continua a cingere il ricordo del generale e della sua eroica armata ha in realtà beneficiato del prestigio e del valore derivanti dalla Description, così come della spettacolare reputazione e dallo sforzo scientifico compiuto dal corpus di artisti e studiosi francesi.

Riuniti inizialmente nella Commission des Sciences et Arts, «primo organo del genere ad unirsi ad una spedizione di conquista militare» ${ }^{8}$, i cosiddetti savants ${ }^{9}$ fornirono per la prima volta, attraverso analisi, osservazioni, scavi e rilevamenti, un bagaglio di conoscenze enormi, di informazioni tratte da fonti autentiche, di dati precisi e puntuali, estremamente preziosi per le future generazioni di studiosi europei. La grande originalità dell'impresa risiedeva dunque proprio nel carattere scientifico dell'esplorazione rigorosa, nella volontà di esportare in una terra dalla storia millenaria e culla per antonomasia di saggezza, ordine e stabilità un sapere nuovo ed enciclopedico, un bagaglio di abilità tecniche e conoscenze scientifiche, secondo il più autentico spirito illuminista della fine del XVIII secolo. Il fine dichiaratamente culturale dell'operazione nasceva dal profondo amore del futuro imperatore per la scienza e le $\operatorname{arti}^{10}$, oltre che dalla consapevolezza che una conquista militare, se unita ad una conquista e appropriazione scientifiche, avrebbe certamente partorito risultati più duraturi nel tempo, assicurando indelebile gloria ai suoi protagonisti. Anche Fourier ${ }^{11}$ nella sua Préface Historique sottolinea che durante la campagna:

Le Général en chef n'avoit point cessé de veiller aux intérêts des sciences. Ce grand objet étoit toujours présent à sa pensée, avant ou après la victoire, soit qu'il dirigeât les opérations militaires, soit qu'il méditât de nouvelles dispositions administratives ou politiques. [...] Les derniers jours qui précédèrent son départ il s'occupoit encore de favoriser les succès des re-

(8) Laure Murat, Nicolas Weil, L’Expédition d'Égypte. Le rêve oriental de Bonaparte, Paris, Gallimard, 1998, pp. 110-111.

(9) Si tratta di un termine dispregiativo utilizzato dai soldati dell'esercito francese in rotta verso l'Egitto, per indicare il gruppo di artisti, ingegneri, architetti, scienziati e studiosi in genere che avrebbero accompagnato la spedizione, ma la cui presenza era loro particolarmente invisa. Il rapporto tra savants e militari fu infatti da subito piuttosto conflittuale, poiché i soldati vedevano negli studiosi la principale causa dei pericoli a cui erano costantemente esposti. Inoltre i savants, pur non potendo svolgere attività militari in senso stretto, spesso assumevano veri e propri incarichi in qualità di esperti e consiglieri in questioni militari.

(10) Per ammissione dello stesso Napoleone, il suo insegnante di matematica durante la scuola elementare, padre Petrault, «développa son penchant pour les sciences». A Parigi, negli anni 1784-85 mentre frequentava l'École des cadets-gentilshommes, Bonaparte aveva la reputazione di "meilleur mathématicien de l'École". Da notare peraltro che il criterio di selezione dei candidati di scuole militari era per l'appunto di tipo scientifico. Cfr. Nicole et JeAn DhOMBres, Naissance d'un nouveau pouvoir: sciences et savants en France. 1793-1824, Paris, Payot, 1989, pp. 647-648. Non dimentichiamo inoltre che la strada di Napoleone si incrociò presto con quella degli intellettuali francesi, i cosiddetti idéologues, alla ricerca, questi ultimi, del- l'appoggio di un potere esecutivo adeguatamente forte anche a livello militare e in linea con i principi repubblicani da loro professati. Come ha scritto Moravia, «nelle alte sfere dell'esercito nessuno poteva vantare credenziali migliori del generale Napoleone Bonaparte». D'altra parte, lo stesso $\mathrm{Na}$ poleone aveva più volte manifestato una spiccata simpatia per le personalità facenti parte del gruppo degli idéologues (come dimostra l'amicizia intessuta con Volney) e un evidente interesse per le idee filosofico-scientifiche e letterarie da essi propugnate. Inoltre, un'assidua frequentazione gli valse l'ambita e prestigiosa nomina a membro dell'Institut de France nel 1797. Da quel momento ebbe inizio un rapporto lungo, ma a tratti estremamente drammatico e travagliato, che caratterizzerà profondamente l'età napoleonica. Cfr. Sergio Moravia, La "raison" $e$ il potere. Il confronto tra tradizione illuminista e regime napoleonico, in DaniELA GaLLINGani, (a cura di), Napoleone e gli intellettuali. Dotti e "bommes de lettres" nell'Europa napoleonica, Bologna, il Mulino, 1996, pp. 37-39.

(11) Autore della famosa Théorie analytique de la Chaleur (1822), Jean-Baptiste Joseph Fourier (1768-1830) è conosciuto per essere stato uno dei più insigni matematiçi e fisici teorici francesi. Professore di analisi all'École Polytechnique di Parigi, fu al seguito di Napoleone durante la Campagna d'Egitto. Nel 1802 fu nominato prefetto dell'Isère, nel 1817 membro dell'Académie des Sciences e nel 1826 dell'Académie de France. 
cherches savantes, en donnant à l'Académie qu'il avoit formée, les moyens de parcourir les provinces méridionales de l'Égypte, et d'en observer les merveilles avec sécuritét ${ }^{12}$.

Peraltro l'idea di creare il binomio contingente militare-commissione di studiosi non era nuovo all'esperienza francese: già a partire dal III anno della Repubblica, un piccolo gruppo di pittori e botanici era stato incaricato di operare sul Reno e nei Paesi Bassi, con il compito di raccogliere quadri e sculture a profitto dei musei francesi, e di compiere studi nell'ambito della storia naturale. Ancora, durante la Campagna d'Italia le requisizioni artistiche furono numerosissime e una vera e propria Commissione di Scienza e Arti d'Italia fu fondata a tale scopo ${ }^{13}$. Infine, lo stesso Bonaparte, dopo il trionfale ritorno in patria a seguito della Campagna d'Italia il 15 frimaio Anno VI (5 dicembre 1797), fu nominato membro della prima classe (Scienze fisiche e matematiche) dell'Institut de France, sul modello del quale, l'anno successivo (20-21 agosto 1798) al Cairo verrà fondato l'Institut d'Égypte ${ }^{14}$.

Il corpo letterario e scientifico formatosi in Egitto, e comprendente 167 membri, aveva come scopo coltivare e perfezionare tutte le conoscenze teoriche e moltiplicarne le applicazioni pratiche. La Description, il cui obiettivo primario era raccogliere e presentare i risultati delle principali ricerche intraprese durante la spedizione, ha lasciato alla posterità un'enorme quantità di materiale concernente l'antichità, lo stato attuale, la storia naturale e la geografia dell'Egitto; in sostanza, tutti gli elementi utili allo studio di questo paese. Per il suo approccio sistematico e per il suo monumentale carattere enciclopedico, l'opera costituisce dunque un vero e proprio inventario ragionato di tipo storico-culturale, naturalistico e archeologico, ciò che oggi viene definito un archivio di informazioni. La Description è frutto dello sforzo congiunto e parallelo dei più validi e illustri studiosi e scienziati del panorama francese di fine Settecento, uomini di grande levatura culturale e dagli indiscussi talenti, che hanno apportato alla madrepatria non solo vantaggi di ordine politico-commerciale, ma altresì scientifico e artistico. Questo fortunato e prolifico concorso di abilità non ha precedenti nella storia dei viaggi letterari. Le scoperte che ne sono scaturite hanno dunque un carattere di unicità, portando in sé il tratto di una preziosa collaborazione, spesso cementata anche da una solida amicizia tra i membri della spedizione stessa, e da una comunanza di interessi e vedute, capaci di rendere la lontananza dalla patria e i pericoli connessi alla precarietà della situazione politico-militare meno oppressivi ${ }^{15}$.

La spedizione d'Egitto è considerata uno degli eventi più significativi della fine del XVIII secolo. Dice a questo proposito Fourier:

Pour réussir dans une entreprise aussi hardie, il ne falloit pas moins qu'une armée composée d'hommes d'élite, conduite et commandée par des généraux dont les talens et l'habilité avoient toujours su maitriser la victoire. Indépendamment des grands avantages que cette conquête pouvoit procurer sous les rapports politiques et commerciaux, il y en avoit un bien considérable sous le rapport des sciences et des arts: on sait que l'Égypte a été leur berceau, et qu'il existe beaucoup des monumens de son ancienne splendeur; mais ils étoient peu connus, et le sol sur lequel ils reposent ne l'étoit pas davantage. [...] Il étoit réservé à la France de conquérir ces contrées célèbres, de les décrire avec fidélité, et de donner cette impulsion qui depuis a fait faire encore des recherches et des découvertes importantes. Pour atteindre à ce but, des savans illustres de

(12) Description de l'Égypte, Préface et explication des planches, 1809, Préface Historique, p. LXI.

(13) Di essa facevano parte due insigni studiosi, destinati a legare per sempre il proprio nome alla campagna napoleonica in Egitto: il matematico Gaspard Monge e il chimico Claude-Louis Berthollet. Cfr. Fernand Beaucour, Yves Laissus, Chantal
Orgogozo, La découverte de l'Égypte, Paris, Flammarion, 1989 , p. 69.

(14) Cfr. Olivier Nolin, Bonaparte et les savants français en Égypte. 1798-1801. Paris, Éditions Mille et Une Nuits, 1998, pp. 70-74.

(15) Cfr. Description de l'Égypte, Préface et explication des planches, 1809, «Avertissement», p. 1. 
tous les genres, qui n'avoient rien à ajouter à leur réputation, ne craignirent point de quitter leurs foyers, des partager les périls et les fatigues de l'armée, guidée par des chefs que leurs lumières mettoient à même d'allier l'amour des sciences avec la gloire des armes. Cet exemple fut suivi par des artistes distingués, et par une jeunesse éclairée, pleine d'ardeur pour les découvertes ${ }^{16}$. Ce grand ouvrage intéresse la gloire de notre patrie: on le doit aux efforts de ses guerriers, il tire son origine de l'union des sciences et des armes: il est le témoignage et le fruit de leur alliance ${ }^{17}$.

$$
* * *
$$

L'esperienza dei savants francesi rappresenta un punto di rottura con i precedenti resoconti di viaggio, spesso fantasiosi, arbitrari o personali, nutriti di clichés e stereotipi che tratteggiavano un Egitto mitizzato e fantastico, oppure semplicemente imprecisi e incompleti per la mancanza di informazioni e dati oggettivi e inoppugnabili. Per questo, benché un approccio storiografico sia necessario per comprendere appieno il contesto politico della spedizione (le cui origini affondano ben prima di quel tumultuoso finire del XVIII secolo) è di particolare interesse esaminare attentamente i legami e il fascino esercitato dal nascente filone della letteratura di viaggio, che pone come obiettivo proprio quella regione dell'Oriente magico e trasognato, meta di numerosi, avventurosi itinerari.

Sarà pertanto opportuno vagliare non solo il contributo che la Description e la spedizione napoleonica hanno fornito nell'ambito della letteratura di viaggio del XIX secolo, i cui impulsi si coloreranno presto di istanze turistiche, ma altresì valutare la portata dell'opera alla luce degli apporti che essa ha ricevuto da coloro che l'hanno preceduta in ordine di tempo, avventurandosi in un mondo ancora semisconosciuto, capace di stimolare all'esplorazione le menti dei più fervidi viaggiatori.

Esaminando i 9 volumi di testo che costituiscono la Description, ci si accorge ben presto che il viaggio attraverso le pagine dotte dell'opera è accompagnato dalla presenza costante e indispensabile dei viaggiatori che hanno preceduto in Egitto i savants francesi per i più svariati motivi di studio e ricerca. Ogni singola parte dell'opera $(A n$ tiquités, État Moderne e Histoire Naturelle) è pervasa dalla loro eco e gli studiosi francesi hanno saputo coglierla, arricchendo quest'importante eredità di nuovi orizzonti e prospettive scientifiche sino a darle la forma di un'opera enciclopedica unica nel suo genere. Ha reso possibile questo trapasso e arricchimento di conoscenze proprio la volontà dei viaggiatori del passato di mettere a disposizione della collettività un insieme di osservazioni ed esperienze che non fossero unicamente espressione di singole individualità, ma rappresentassero altresì un'esigenza di certezza e allargamento dei campi del sapere. Quasi si percepisse il dovere morale di colmare le lacune del passato con saperi polivalenti e nuove conoscenze nelle discipline in evoluzione ${ }^{18}$. Gli esponenti di questa prolifica letteratura di viaggio presentano una vasta e variegata produzione che, secondo Henry Laurens ${ }^{19}$, può essere distinta in differenti categorie:

- I resoconti derivanti dalle prime missioni archeologiche sino a giungere ai viaggi di Paul Lucas;

- I viaggi di carattere religioso, entro cui confluiscono le esperienze di gesuiti o cappuccini, spinti dal fervore della Contro Riforma. Rientrano senz'altro in questa partizione le relazioni dei padri Sicard e Vansleb;

(16) Pierre Jacotin, Mémoire sur la construction de la carte d'Égypte, in Description de l'Égypte, État moderne, vol. II, $2^{\circ}$ parte, 1822 , p. 1.

(17) Description de l'Égypte, Préface et explication des planches, 1809, Préface Historique, p. LXXXVIII.

(18) Nicole Hafid-Martin, Voyage et con- naissance au tournant des Lumières. 1780-1820, Oxford, Voltaire Foundation, 1995, p. 235.

(19) HenRY LauRENs, Les origines intellectuelles de l'Expédition d'Égypte: l'orientalisme islamisant en France (1698-1798), Istanbul, Ed. Isis, 1987, pp. 16-17. 
- Memorie e resoconti di viaggio di ufficiali di marina (Norden) o di diplomatici e agenti consolari (Benoît de Maillet), che avessero lo scopo di coltivare fiorenti relazioni con l'Oriente;

- Memorie politiche e di ispezione territoriale volte a valutare l'opportunità di un'espansione europea in Oriente, quali la relazione di padre Coppin ${ }^{20}$ verso la metà del Seicento o le memorie del barone de $\operatorname{Tott}^{21}$;

- Infine, i primi resoconti di memorie scientifiche che costituiscono una naturale evoluzione rispetto alle prime missione archeologiche e che muteranno il volto della letteratura di viaggio, a partire dall'avventura del tedesco Niebuhr, unico sopravvissuto della spedizione danese in Arabia tra 1761 e 1767. Queste esperienze costituiscono a buon diritto i veri e propri antesignani della spedizione francese in Egitto.

Come precedentemente accennato, la Description è costellata in tutta la sua imponente estensione di nomi di dotti studiosi, viaggiatori, orientalisti e naturalisti, le cui opere rientrano in questa partizione. Lo scorrere paziente delle pagine di memorie, descrizioni e osservazioni della Description consente altresì di chiarire come le testimonianze precedenti di viaggiatori in Egitto siano state accolte dai savants e come, in numerosi casi, esse abbiano finito per costituire un punto di riferimento obbligato per i membri della Commission e dell'Institut.

La Description è riconducibile ad un fenomeno emblematico nella società civilizzata dei Lumi di fine Settecento, ed essa costituisce per tale motivo una sorta di spartiacque nell'ambito della letteratura di viaggio, rispetto alle esperienze precedenti di cui comunque si nutre. Queste ultime sono spesso influenzate da una configurazione "esotizzante" dell'Oriente in cui esso viene messo in scena come rêverie fantastica e metafora della realtà. La tendenza che caratterizzava i visitatori e viaggiatori in Oriente, e in Egitto nella fattispecie, era riconducibile ad un sistema di ristrutturazione della realtà percepita in chiave romantica, secondo preconcetti imperanti, descrizioni stereotipate e clichés tradizionali: «Cet Orient sera très certainement déformé par la culture, la société de celui qui l'observe et le juge, autrement dit par l'héritage culturel de chaque voyageur et son milieu d'origine» ${ }^{22}$.

L'esigenza per molti era fornire impressioni di viaggio elaborate dall'immaginazione $\mathrm{o}$ in prospettiva fantastica, più che regalare fotografie e scorci di realta ${ }^{23}$. Il potere evocativo della parola veniva poi spesso potenziato dall'immaginario raccolto dai pittori e dagli artisti, che aprivano così uno scorcio su questo mondo meraviglio-

(20) J. Coppin, Relation des voyages faits dans la Turquie, la Thébaïde et la Barbarie contenant des avis politiques qui peuvent servir de lumières aux Rois, Lyon, 1686. Padre Coppin viaggiò in Egitto nel 1638, ma la sua relazione apparve quasi cinquant'anni dopo, posteriormente rispetto ai resoconti di Jean de Thévenot o di padre Vansleb. Il tono aggressivo dell'opera, pur documentata e copiosa, la configura come «un appel à toute la Chrétienté contre les Infidèles, une exhortation lancée à tous les rois catholiques pour provoquer une nouvelle Croisade contre les Turcs, dont le Père Coppin se propose d'indiquer les points faibles et de démasquer l'impuissance») (JEAN-MARIE CARRÉ, Voyageurs et écrivains français en Égypte, vol. I, $2^{\mathrm{a}}$ ed., Le Caire, Institut Français d'Archéologie Orientale du Caire, 1990, pp. 18-19).

(21) BARON DE TOTT, Mémoires sur les Turcs et les Tartares, Amsterdam, 1784. La famosa missione segreta affidata al barone de Tott, inviato nel 1771 in Egitto con l'interprete Venture de Paradis, aveva lo scopo di fornire un resoconto dettagliato sull'Egitto, che ne descrivesse le risorse e ne tracciasse un piano di conquista e penetrazione commerciale. Ciò poteva concretizzarsi attraverso l'apertura di un varco verso le Indie, che toccasse il Mar Rosso, il Canale di Suez e la città di Alessandria.

(22) Yvelise Bernard, L'Orient du XVI ime siècle à travers les récits des voyageurs français: regards portés sur la société musulmane, Paris, L'Harmattan, 1988 , p. 24.

(23) Nadia El Kholy, Romances and Realities of Travellers, in JANET and PAUL STARKEY (a cura di), Unfolding the Orient. Travellers in Egypt and the Near East, Ithaca Press, 2001, pp. 261-273. 
so, riscoprendone la storia passata, la mitologia e i testi sacri ${ }^{24}$. In tal modo, coloro che hanno soggiornato in Egitto hanno fornito a volte documentazione oggettiva, altre volte semplicemente ispirazione evocativa a quanti non hanno mai intrapreso questo viaggio.

La spedizione francese ha avuto il merito di aver sollevato per sempre il velo di ombra e mistero che da secoli circondava l'Egitto, consegnandolo per la prima volta ad un rigoroso e completo studio scientifico, in cui istanze politiche, ideologiche, militari, culturali ed artistiche si sono fuse armoniosamente. Ciò è testimoniato dagli obiettivi stessi della Description de l'Égypte, riassunti ed esposti efficacemente da Fourier nella Préface Historique:

L'intérêt des beaux-arts et de la littérature exigeoit encore une description fidèle et complète des monumens qui ornent, depuis tant de siècles, les rivages du Nil, et font de ce pays le plus riche musée de l'univers. On a mesuré toutes les parties de ces édifices avec une précision rigoureuse, et on a joint aux plans d'architecture les plans topographiques des lieux où les villes anciennes étoient situées; on a représenté, dans des dessins particuliers, les sculptures religieuses, astronomiques ou historiques, qui décorent ces monumens. [...] On a levé un grand nombre de cartes géographiques, qui représentent, d'une manière exacte et détaillée, la situation des côtes et des ports, celle des villes actuelles, des villes anciennes, des villages, des hameaux, ou des autres points remarquables, et le cours du Nil, depuis la cataracte de Syène jusqu'à la Méditerranée. Ce travail est fondé sur des observations astronomiques. Enfin on s'est appliqué à l'examen de toutes les productions naturelles, ou du moins à celui des faits les plus importans ou les moins connus de la zoologie, de la botanique et de la minéralogie.

Les résultats de ces différentes recherches sur l'histoire naturelle et la géographie de l'Égypte, sur ses antiquités et son état moderne, ont été réunis dans un seul ouvrage [qui] a donc pour objet de donner une connoissance exacte et approfondie de l'Égypte; elle présente les vrais élémens de l'étude physique, littéraire et politique d'une des contrées les plus remarquables du globe ${ }^{25}$.

L'Egitto è sempre stato il paese del Vicino Oriente che maggiormente ha attratto e affascinato il mondo occidentale. Un fascino che si è manifestato in numerose direzioni a partire dalle istanze religiose delle crociate, dove l'Egitto si configurava non come meta, ma come passaggio obbligato nell'itinerario che conduceva in Palestina ${ }^{26}$, da quelle artistico-culturali che hanno spinto al viaggio, all'esplorazione e al confronto tra due codici diversi, sino a giungere ai legami con il mondo della politica e del commercio che hanno fatto dell'orientalismo un sistema di sfruttamento dell'Oriente da parte dell'Occidente di tipo colonialista. Ciascuno di questi atteggiamenti ha comunque avuto come denominatore comune l'interesse per l'Oriente nella sua specificità e alterità.

(24) Sophie Monneret, L'Orient des peintres, Paris, Nathan, 1989, pp. 1-2.

(25) Description de l'Égypte, Préface et explication des planches, 1809, Préface Historique, p. VII.

(26) L'Egitto è sempre stato terra di passaggio non solo per i pellegrini cristiani, ma anche per commercianti, artigiani e grandi viaggiatori in generale che da lì raggiungevano la Terra Santa o si imbarcavano alla volta delle Indie e della Cina. La visita all'Egitto, e in modo particolare alla città del Cairo, rappresentava comunque un modo per completare il proprio periplo dei luoghi santi. Il fatto che lo spazio, attualmente compreso tra il Canale di Suez e il Mar Rosso, fosse costituito da una ban- da di deserto montuoso entro cui si riconoscono ampie vallate trasversali praticabili da carovane, spiegherebbe perché l'Egitto sin da tempi remoti sia stato il tramite principale dell'Europa nel commercio con le Indie. Dalla parte dell'Asia, invece, si sviluppava immediatamente una vasta distesa di deserto, percorribile in diversi giorni di cammino, interrotta dall'estendersi della Siria. Cfr. MicheL ROZIÈre, De la géographie comparée et de l'ancien état de la Mer Rouge considérés par rapport au commerce des égyptiens dans les différens âges. Introduction et première partie, in Description de l'Égypte, Antiquités, Mémoires, vol. 1, 1809, p. 129. 
Storicamente è stata la guerra il primo vero impulso ad aver portato gli occidentali ad avventurarsi in Oriente. Spinti dallo zelo religioso e dalla suggestione esercitata dal ricco immaginario biblico, essi hanno abbandonato le proprie terre, poiché si compisse attraverso le crociate il loro destino di vittime o carnefici. Secondo la coscienza medievale il viaggio in Terra Santa era un'esperienza totalizzante, non solo vissuta come spedizione militare, ma come pellegrinaggio che consentiva spesso la partenza con i crociati di donne e bambini ${ }^{27}$.

Ben presto, sopraggiunse la consapevolezza che la «civiltà mediterranea ha avuto come culla l'Africa settentrionale e il Vicino Oriente ${ }^{28}$ : con la sua storia millenaria e un sapere antico e misterioso l'Egitto cominciò a dare impulso anche a viaggi di studio e di esplorazione. Sarà soprattutto il Rinascimento a tentare di affermare l'originalità e il primato della civiltà egizia rispetto a quelle ellenica e latina.

Numerosi sono coloro che hanno scritto in questi secoli dell'Egitto (le relazioni di viaggio cominciano a farsi abbondanti e importanti nel XIV, XV e XVI secolo, e danno conto dell'evoluzione delle mentalità e degli sguardi che hanno come oggetto proprio l'Egitto). Tra essi è possibile rilevare resoconti o diari di viaggio che attestano una reale esperienza nella "terra dei Faraoni" e che dunque hanno lasciato una preziosa testimonianza e un deciso contributo alla delineazione delle conoscenze europee relative a questa terra. Molti altri scrittori, al contrario, si sono abbandonati a racconti puramente nutriti di fantasia e di clichés più o meno codificati, per fissare l'immagine di un Egitto fantastico e immaginato che poco ha, tuttavia, di realistico. Questi ultimi, acutamente classificati da Jeannine Guerin Dalle Mese ${ }^{29}$ come "viaggiatori sedentari”, sono protagonisti di viaggi fittizi ed i loro resoconti attingono direttamente alle descrizioni dei predecessori (Erodoto, Diodoro Siculo, Strabone, Plinio il Vecchio...). Sono i cosiddetti "viaggi da camera", in cui il viaggio simbolico sostituisce il viaggio empirico, che tuttavia nulla perde della propria autenticità e veridicità. Esempio illustre ci viene fornito dall'Itinerarium in Terra Santa di Francesco Petrarca che nel 1358, basandosi sulla Bibbia, su indicazioni di geografi e di autori classici compilò un percorso immaginario. Questo ci porta a pensare che nel Medioevo il viaggio non avesse l'accezione moderna di scoperta ed esplorazione di territori sconosciuti, ma piuttosto di rivisitazione di luoghi già fissati nella memoria collettiva.

I primi veri viaggiatori sono «monaci e uomini di Chiesa inviati spesso in Terra Santa per scacciare i saraceni, liberare i luoghi santi o portare soccorso agli schiavi cristiani e agevolare i pellegrinaggi» ${ }^{30}$, che si diffondono parallelamente all'ideale missionario. I missionari sono i veri esploratori del Medioevo e grazie alla loro cultura hanno potuto lasciare importanti fonti scritte. Ma nondimeno vanno ricordati i laici che, provenienti da tutte le nazioni europee, costituiranno un nutrito gruppo tra XIII e XIV secolo. Gli italiani sono comunque i più numerosi, soprattutto coloro che provengono dalle grandi Repubbliche marinare e da città che vantano secolari tradizioni nell'ambito del commercio internazionale. Purtroppo i più si sono persi nell'oblio perché non si sono curati di lasciare traccia dei propri spostamenti o di affidare la loro memoria alla pagina scritta. In generale, nel corso del XII e XIII secolo una serie di

(27) La bibliografia relativa ai viaggi nel Medioevo è estremamente vasta e articolata. Tra i titoli più significativi si ricordano: N. OHLER, I viaggi nel Medioevo, Milano, Garzanti, 1988, R. STOPAnI, Le vie di pellegrinaggio del Medioevo. Gli itinerari per Roma, Gerusalemme, Compostella, Firenze, Le Lettere, 1991, M. S. MazzI, Oltre l'orizzonte. In viaggio nel Medioevo, Torino, Gribaudo-Paravia, 1997, F. CARDINI, In Terrasanta. Pellegrini italiani fra Medioevo e prima età moderna, Bologna, il Mulino, 2002.

(28) Calvesi Maurizio, Il mito dell'Egitto nel Rinascimento. Pinturicchio, Piero di Cosimo, Giorgione, Francesco Colonna, Firenze, Giunti, 1988, p. 5.

(29) Jeannine Guerin Dalle Mese, Égypte: ia mémoire et le rêve. Itinéraire d'un voyage, 13201601, Firenze, L. Olschki, 1991, pp. 13-14.

(30) Ibid., p. 17. 
avvenimenti e circostanze, quali le crociate, la rinascita intellettuale e l'impulso dato agli studi creano le condizioni favorevoli alle grandi esplorazioni medievali ${ }^{31}$.

Già nel 1202 ci lascia traccia della sua esperienza in Egitto il pisano Leonardo Fibonacci che scrive il Liber abaci, un'opera pratica indirizzata a mercanti.

Nel XIV secolo un laico, Marino Sanudo, detto Torsello, scrive il Liber secretorum fidelium Crucis super Terrae Sanctae recuperatione et conservatione quo et Terrae Sanctae Historia ab origine et eiusdem vicinarumque provinciarum geographica descriptio continetur, in cui sono rivolti utili consigli al Papa e alla Chiesa per la riconquista della Terra Santa: l'Egitto, infatti, canale di accesso alla Palestina, era un passaggio obbligato anche per gli eserciti impegnati nelle crociate.

Sempre nel XIV secolo si ha il primo testo in volgare ad opera del frate Niccolò da Poggibonsi: il Libro d'Oltramare (1346-1350). Questo sarà seguito, verso la fine del secolo, da un'accurata relazione in lingua volgare del proprio viaggio in Egitto di Leonardo Frescobaldi, dal titolo Viaggio di Lionardo di Niccolò Frescobaldi fiorentino in Egitto e in Terra Santa (1384).

Accanto ai viaggiatori religiosi vi sono poi laici, ambasciatori inviati in missione, diplomatici, oratori, mercanti e umanisti. Il caso più famoso è quello di Ciriaco d'Ancona $^{32}$ a metà del XV secolo.

Molteplici sono le testimonianze pervenuteci dal XVI secolo, rappresentative delle modalità del pensiero umanista; in esse possiamo scorgere il cambiamento della prospettiva europea nei confronti dell'Egitto come possibile meta o tappa di viaggio. Se precedentemente l'Egitto si configurava come luogo di passaggio, sia per i pellegrini diretti in Palestina, sia per mercanti e commercianti, in quanto situato sulla via per le Indie e la Persia, nel XVI secolo esso comincia a divenire oggetto d'interesse di per sé, in quanto paese ancora sconosciuto, luogo pieno di meraviglie, ricco di mistero e di curiosità. Prende a circolare un'immagine piuttosto favolosa, veicolata dai resoconti di viaggiatori e da fonti di letteratura popolare che si occupano di "mirabilia". A partire dalla metà del XVI secolo l'Egitto non sarà più solo una destinazione occasionale, ma la consapevole meta di un itinerario che il viaggiatore consacra interamente a questa terra, all'insegna dell'avventura, della scoperta e dello studio. Abbondano le descrizioni geografiche del territorio, allora limitatamente conosciuto sia nel tempo, sia nello spazio: la storia nota è per lo più quella inerente le crociate, e solo pochi si spingono a studiare il periodo greco e romano, o addirittura l'antichità egizia, mentre i luoghi frequentati si riducono al triangolo Il Cairo-Damietta-Alessandria e al deserto che conduce al Sinai ${ }^{33}$. Ma l'interesse riguarda anche la storia naturale, la botanica, la zoologia, la riscoperta di preziose conoscenze di cui era considerato depositario l'antico Egitto dei Faraoni, come la geometria, l'astrologia, la cosmografia e la scienza in genere ${ }^{34}$.

(31) Jean Paul Roux, Gli esploratori nel Medioevo, Milano, Garzanti, 1990, p. 41.

(32) Ciriaco, appartenente a una famiglia di mercanti, si imbarcò per la prima volta per raggiungere Alessandria (ma numerosi saranno i suoi viaggi in Egitto) all'età di vent'anni, sulla nave di un parente per prestare servizio come scriba minor. Cfr. Jeannine Guerin Dalle Mese, op. cit., p. 20. Per un'analisi più approfondita della poliedrica figura di Ciriaco d'Ancona si veda in generale J. Colin, Cyriaque d'Ancône. Le voyageur, le marchand, l'bumaniste. Paris, Maloine, 1981.

(33) Per molto tempo il punto estremo dei viaggi verso sud fu rappresentato dalla prima cataratta del Nilo, secondo l'itinerario di Erodoto. L'unica esplorazione ad aver presumibilmente superato quel limite sembra sia stata intrapresa da Diogene, marinaio greco, nel 50 a. C. Cfr. Jean Paul Roux, op. cit., p. 16.

(34) Alla metà del XVI secolo molti viaggiatori sono animati da una profonda curiosità per la storia naturale. Numerosi medici si recano in Egitto e accumulano una quantità di osservazioni che faranno poi confluire nei propri resoconti di viaggio, mentre altri, appassionati di archeologia, risaliranno il corso del Nilo alla ricerca di templi faraonici. Non si tratta più, dunque, di pellegrini o militanti cattolici incaricati di fornire alla Chiesa utili informazioni su come conquistare la Palestina, ma di laici il cui ambito di ricerca investe ora an- 
Ripercorrendo le orme degli antichi, numerosi sono coloro che nel corso del XVI secolo cercheranno la chiave di questa civiltà perduta gettando le basi che saranno poi completate attraverso la spedizione d'Egitto. La Description comincia infatti a dare conto delle esperienze del passato proprio a partire dal Cinquecento, dopo aver fatto un salto nel tempo che l'aveva inizialmente portata sulle orme degli antichi - Erodoto, Diodoro Siculo, Strabone, Plinio il Vecchio, Plutarco, Apollonio Rodio, Teocrito e altri - guide e riferimenti obbligati nel corso delle trattazioni dotte dei membri della Commission e dell'Institut. Le pagine dell'opera cominciano così ad annoverare i nomi di studiosi e viaggiatori cinquecenteschi: Pierre Belon du Mans ${ }^{35}$, André Thevet ${ }^{36}$, Jean Palerne ${ }^{37}$, Jacques de Villamont ${ }^{38}$ e

che la sfera del "profano". Tra i numerosi esempi citiamo le seguenti opere del XVI secolo: LODOVICO DE VARTHEMA, Ludovicii patritii Romani novum itinerarium Aethiopiae Aegipti utriusque Arabiae Persides Siriae ac Indiae intra et extra Gangem, Milano, 1510, Filippo Pigafetta, Relazione o viaggio del signor Filippo Pigafetta nobile vicentino, d'intorno al viaggio nello Egitto, nell'Arabia, nel Mar Rosso et nel Monte Sinai (manoscritto autografo della fine del XVI sec., circa 1576-77). A Pigafetta si deve tra l'altro il disegno della "Grande Carta d'Africa" in cui appare il progetto del Canale di Suez.

(35) Pierre Belon du Mans, Les observations de plusieurs singularitez et choses mémorables, trouvées en Grèce, Asie, Judée, Égypte, Arabie et autres pays estranges, Paris, 1553. Belon, una delle personalità più rappresentative e poliedriche del XVI secolo, nacque nel 1516 o 1517. La mancanza di informazioni relative ai primi anni di vita lascia comunque presagire che egli appartenesse ad una famiglia di modeste origini. Passò parte della sua adolescenza in Bretagna consacrandosi alla raccolta erboristica, passione che condividerà con il vescovo di Mans, suo amico, protettore e finanziatore degli studi a Parigi. Da Parigi, Belon si recò in Sassonia, a Wittenberg, nel 1540 per seguire all'università il corso del celebre Valerius Cordus, divenuto successivamente suo grande amico, e perito tragicamente a Roma dopo una caduta da cavallo. Dopo alcune peregrinazioni in Germania, Lussemburgo e Italia per raccogliere fossili e studiare il suolo e le sue produzioni, Belon ritornò in Francia per dedicarsi completamente all'erboristeria. Desideroso di poter constatare di persona le osservazioni degli antichi relativamente alle specie di piante raccolte, seguì poi l'ambasciatore Gabriel d'Aramon inviato presso la Porta nel 1547, e viaggiò in Oriente sino al 1549. La sua avventurosa e laboriosa vita fu interrotta tragicamente dall'aggressione di un criminale, durante l'attraversamento del Bois de Boulogne, nel 1564. Cfr. Yvelise Bernard, L'Orient du XVI ime siècle à travers les récits des voyageurs français: regards portés sur la société musulmane, Paris, L'Harmattan,1988, pp. 29-31.

(36) AnDRÉ ThÈvet, Cosmographie du Levant, Lyon, Jean de Tournes et Guillaume Gazeau, 1556. Nato probabilmente ad Angoulême nei primi anni del XVI secolo, prese l'abito dei Cordeliers e completò gli studi di teologia, benché una passione interiore lo spingesse verso le scienze profane. Dopo molti patimenti, riuscì infatti ad ottenere dai suoi superiori l'autorizzazione a viaggiare, in
Italia, Grecia, Brasile e Oriente. Nel 1558 ottenne la secolarizzazione e la nomina a elemosiniere di Caterina de Medici, oltre alla carica di storiografo e cosmografo del Re. Morì a Parigi nel 1592, all'età di 88 anni. Cfr. Yvelise Bernard, op. cit., pp. 3839. Benché le cento pagine che Thévet consacra all'Egitto pretendano di assumere la forma di un trattato scientifico, in esse è possibile cogliere numerosi errori e interpretazioni fantasiose, che gli hanno spesso procurato accuse di ingenuità $\mathrm{e}$ credulità eccessive. Lettore insaziabile e dotato di una memoria straordinaria, era in grado di raccogliere velocemente informazioni su un soggetto e di parlarne con estrema facilità. Insieme alla relazione di Pierre Belon, quella di Thévet è comunque considerata opera fondamentale per il contributo dato all'allargamento delle conoscenze relative all'Egitto nel xvi secolo.

(37) Jean Palerne, Pérégrinations de S. Jean Palerne, Forézien... où il est traicté de plusieurs singularitez E antiquités remarquées ès provinces d'Egypte, Arabie déserte et pierreuse...etc., Lyon, Jean Pillehotte, 1606. Pare che Jean Palerne sia nato nei pressi di Saint-Étienne-en-Forez verso il 1557. Figlio di un medico, fece solidi studi, tanto che lo ritroviamo segretario del Duca d'Alençon, fratello del re Enrico III, con il quale viaggiò attraverso Francia e Inghilterra. Visitò in seguito la Spagna e si recò in viaggio in Oriente dal 1581 al 1583. Non abbiamo purtroppo notizie successive al suo ritorno. Morì prematuramente nel 1592 all'età di 35 anni. Cfr. Yvelise Bernard, op. cit., pp. 43-44.

(38) SR DE Villamont, Les voyages du sieur de Villamont, divisez en trois livres, ...etc. Et au troisième est la description de Syrie, de Damas, Phénicie, Égypte, Damiette, du grand Caire, de Babylone, des anciennes Pyramides et Mommies..., éd. par Gilles Baudhuin, 1595-1598. Anche nel caso di Villamont, poche sono le notizie biografiche a disposizione; la data della morte dovrebbe comunque attestarsi intorno al 1629 . Un'insaziabile curiosità lo portò a viaggiare in Oriente, dove visitò Siria, Palestina, Egitto, Turchia e Monte Sinai, facendo anche numerose escursioni che lo condussero fuori dall'itinerario, in Italia e a Cipro. Il successo della sua opera fu tale da spingere gli editori a ben 26 riedizioni dei Voyages tra 1595 e 1615 . Nel terzo libro due capitoli sono consacrati alla città del Cairo, da lui ritenuta immensa e inestricabile con le sue innumerevoli vie, e alla visita delle piramidi e delle mummie. A tal proposito, la sua descrizione delle piramidi e della sfinge non può essere presa a 
Leone l'Africano ${ }^{39}$. Tra essi spicca senz'altro la figura di Pierre Belon, il cui genio e la cui straordinaria capacità di osservazione e analisi ne faranno uno dei più grandi naturalisti e botanici del suo tempo, precursore dell'anatomia comparata e primo ad aver tentato una classificazione delle specie vegetali di cui si servirà Linneo due secoli più tardi. Le sue Observations, afferma Jean-Marie Carrée ${ }^{40}$, pur non avendo un grande valore letterario di per sé, sono ammirevoli per la copiosità e minuziosità dei dettagli riportati, che contribuiscono così a renderla la raccolta in lingua francese sul Vicino Oriente più importante e autorevole dell'epoca. All'interno della Description Belon viene citato numerose volte, sia entro le dotte disquisizioni di natura archeologica e geografica dei savants, sia, ovviamente, entro i volumi di Histoire Naturelle. Ad esempio, in relazione alla Grande Piramide di Cheope, Girard ${ }^{41}$ afferma che Belon è stato il primo viaggiatore moderno ad occuparsi della misurazione della base, giungendo ad un risultato di 334 passi, misura peraltro coincidente con quella addotta anche da Pietro della Valle. Oppure, per quanto riguarda lo studio dei laghi del Basso Egitto, ed in particolare il Lago Mareotis, che all'epoca della spedizione francese risultava ormai essere un bacino secco, Le Père ${ }^{42}$ si serve della testimonianza di Belon, così come di altri viaggiatori, tra cui anche Villamont e Thévenot, per avvalorare la tesi secondo cui il disseccamento del bacino avrebbe avuto luogo alla fine del Seicento o all'inizio del Settecento, mentre in precedenza il lago e gli antichi canali che vi affluivano dovevano esistere ancora. Infine, non potevano mancare entro la Description i contributi di Belon alle scienze naturalistiche, annoverati nella specifica partizione ad esse dedicata. Così, possiamo leggere come Geoffroy-Saint-Hilaire ${ }^{43}$ e Savigny ${ }^{44}$ abbiano consultato le Observations del naturalista francese per confrontare la nomenclatura di specie di pesci o uccelli attraverso un'analisi comparata.

Tra XVII e XVIII secolo cominciano a profilarsi all'orizzonte nuove esperienze, non più di natura religiosa, come i tradizionali pellegrinaggi, poiché nuove sono le motivazioni alla base del viaggio: relazioni diplomatiche, missioni commerciali o di ricerca scientifica, sino a giungere nei due secoli successivi, e soprattutto nell'Ottocento, a veri e propri viaggi "letterari" di scrittori desiderosi di cogliere in Egitto visioni, temi, atmosfere da far confluire nelle proprie opere. Come afferma Gianni Guadalupi $^{45}$, «incantesimi piramidali e nilotici facevano da cornice a una dolce vita che ammaliava l'europeo; singhiozzi soffocati di nostalgia trapelano dalle pagine di

modello di esattezza e precisione. Il merito riconosciutogli è tuttavia quello di essere stato il primo e forse unico viaggiatore del XVI e XVII secolo ad aver situato correttamente Menphi, celebre capitale del Regno Antico. Cfr. Yvelise Bernard, op. cit., p. 45 e JEAN-Marie Carrè, op. cit., 1990, pp. 9-11.

(39) Johannes LeO Africanus, Descriptionum Africae rerumque memorabilium liber primus, 1559. Leone Africano fu invitato nel 1517 presso il sultano Selim I, che risiedeva in Egitto nel momento in cui i mamelucchi furono scacciati dagli ottomani. Non è sicuro che egli abbia percorso l'Egitto in tutta la sua estensione, poiché non fa mai, curiosamente, riferimento a Louqsor o Karnak. Tuttavia, Jomard lo menzionerà nella Description (Description de Syène et des cataractes, Antiquités, Descriptions, vol. I, 1809, p. 12) parlando dei dintorni di Syène, e nello specifico di una grande vallata che all'epoca della spedizione risultava ricoperta dalle sabbie, ma un tempo, secondo quanto confermato dalla descrizione di Leone l'Africano appunto, doveva essere fertile e ben coltivata, presumibilmente a grano.
(40) JEAN-MARIE CARRÈ, op. cit., p. 5.

(41) P. S. GIRard, Preuves de l'antiquité de la coudée d'Éléphantine, tirée de sa division en sept parties, et de son emploi dans les pyramides, in Description de l'Égypte, Antiquités, Mémoires, vol. 1, 1809, p. 24.

(42) Gratien Le Père, Extrait d'un mémoire sur les lacs et les déserts de la Basse Égypte, in Description de l'Égypte, Etat moderne, vol. II, Première partie, 1812, p. 470.

(43) Étienne Geoffroy-Saint-Hilaire, Histoire naturelle des poissons du Nil, in Description de l'Égypte, Histoire Naturelle, vol. I, A Paris, Imprimérie Royale, 1809 , p. 39.

(44) Jules-CÉSAR SAVIGNY, Système des oiseaux de l'Égypte et de la Syrie, in Description de l'Égypte, Histoire Naturelle, vol. I, A Paris, Imprimérie Royale, 1809 , p. 73.

(45) Gianni Guadalupi (a cura di), Orienti. Viaggiatori scrittori dell'Ottocento, Milano, Feltrinelli, 1989 , p. 223. 
tutti i reduci dall'esperienza egiziana». In tal modo vengono formandosi altrettante nuove figure di viaggiatori: accanto agli uomini di fede e spada, uomini di legge e della diplomazia, uomini di scienza e di lettere ${ }^{46}$.

La seconda metà del XVII e il XVIII secolo sono considerati i secoli orientalisti per eccellenza: l'interesse filantropico e di evangelizzazione di molti missionari cappuccini e gesuiti, i crescenti interessi scientifici ed economici per la realizzazione di carte geografiche da usare come strumenti di penetrazione commerciale nei nuovi mercati, le curiosità antropologiche legate allo studio delle culture e tradizioni di popoli lontani ed infine la prolifica letteratura di viaggio sfociarono in una sempre più intensa attività di esplorazione da parte degli europei che arricchì l'immagine dell'Oriente attraverso un'attenta riscoperta artistica e culturale ${ }^{47}$.

Ciò che contribuì in maniera determinante allo spostamento dell'attenzione occidentale in Oriente fu il sopraggiungere di condizioni politico-diplomatiche che rendessero il viaggio più sicuro per l'incolumità del singolo (tutto ciò fu possibile a partire dalle capitolazioni concluse nel 1535 tra Solimano il Magnifico e Francesco I) ${ }^{48}$, oltre che le sempre più fiorenti relazioni commerciali tra Oriente e Occidente. Così, circa un secolo dopo, nella Francia del Re Sole si intrattenevano da tempo rapporti privilegiati con la Porta. Nel 1669, ad esempio, Luigi XIV inviò a Costantinopoli il marchese di Nointel, che nel 1673 ottenne il rinnovamento del trattato di regolamentazione del traffico doganale ${ }^{49}$. Non dobbiamo però dimenticare che il viaggiatore occidentale non godeva totalmente di libera circolazione nelle province ottomane, come peraltro continuerà a testimoniare il conte Volney più di un secolo dopo nel corso del suo soggiorno egiziano:

Chez nous, les voyages sont des promenades agréables; là, ils sont des travaux pénibles et dangereux. Ils sont tels sur-tout pour les Européens, qu'un peuple superstitieux s'opiniâtre à regarder comme des forciers qui viennent enlever par magie des trésors gardés sous les ruines par des Génies. Cette opinion ridicule, mais enracinée, jointe à l'état de guerre et de trouble habituel, ôte toute sûreté et s'oppose à toute découverte. On ne peut s'écarter seul dans les terres; $[\ldots]$ on est donc borné aux rivages du fleuve et à une route connue de tout le monde; et cette marche n'apprend rien de neuf ${ }^{50}$.

(46) Yvelise Bernard, op. cit., pp. 368-369.

(47) La generale "ignoranza" occidentale nei confronti dell'Oriente fino a circa la metà del XVII secolo è forse figlia dello spirito tipicamente secentesco, che si nutriva filosoficamente della conoscenza del sé, dell'Io, più che abbandonarsi all'indagine della diversità etnografica e geografica. Cfr. JEANMARIE CARRE, op. cit., introduzione, p. XXV.

Al contrario, come ha ben chiarito Sarga Moussa (SARGa Moussa, La relation orientale. Enquête sur la communication dans les récits de voyage en Orient (1811-1861), Paris, Klincksiek, 1995, p. 8), lo studio dell'orientalismo nel xIX secolo, che qui raggiunge il suo apice dopo aver cominciato a delinearsi nettamente a partire dalla seconda metà del XVII secolo, non è casuale, ma trova la sua logica storica nell'essenza stessa del romanticismo che, rivelando la crisi d'identità dell'Io, spinge al confronto con culture diverse e all'incontro con l'alterità.

(48) Il trattato siglato nel 1535 tra Francia e Impero Ottomano, mentre regnavano Francesco I e Solimano II, costituisce il primo esempio storico di capitolazione. Il regime delle capitolazioni prevedeva il riconoscimento di alcuni privilegi (man mano più estesi) e successivamente la concessione (unilaterale e revocabile) degli stessi da parte del sovrano, ma non si configurava come un trattato formale. In base a questo regime allo straniero in genere venivano garantiti il completo rispetto della persona e dei beni, l'inviolabilità del domicilio, la libertà di commercio, di transito e di religione, e l'esenzione dalle imposte. Inoltre, lo straniero era sottratto alla giurisdizione delle autorità locali e sottoposto a quella del proprio console; in pratica veniva applicato l'antico principio della personalità della legge e non quello della territorialità. Il trattato del 1535 , rinnovato in seguito una decina di volte, costituì un modello per tutti quelli che furono poi stipulati non solo dalla Francia, ma da tutti gli altri paesi europei, Italia compresa.

(49) Nointel fu accompagnato in questo viaggio dai pittori Jacques Carrey e Jean-Baptiste Vanmour. Quest'ultimo si installò presso la corte del Gran Sultano a Costantinopoli svolgendo un ruolo fondamentale nell'influenzare le tendenze stilistiche dell'epoca e ispirando così numerosi artisti orientalisti. Cfr. Sophie Monneret, op. cit., p. 108.

(50) Constantin-François Volney, Voyage en Syrie et en Égypte pendant les années 1783, 1784 et 1785, Paris, Volland, 1787, 2 voll. Edizione consultata, Paris, Volland-Desenne, 1789, p. 12. 
L'itinerario continuava ad essere imposto dalle autorità sempre per questioni di sicurezza $^{51}$, e non era mai solitario poiché necessitava, per esigenze linguistiche, della presenza costante di un interprete o drogman ${ }^{52}$. Inoltre, era spesso auspicabile per il viaggiatore ricorrere al travestimento e all'abbigliamento all'orientale per evitare di attirare gli sguardi ostili dei musulmani, o rischiare di essere scambiato per un trafugatore di tombe e cacciatore di tesori ${ }^{53}$.

Uno degli oggetti di studio privilegiati cominciò così ad essere quella parte del Mediterraneo orientale che, sotto il dominio dell'Impero Ottomano a partire dal 1517, era più nota agli europei con il termine evocativo di Levante. Legato prevalentemente nel Seicento all'ambito commerciale, a causa delle sempre più intense relazioni e transazioni economiche con la Porta, nel Settecento il concetto di Levante, grazie soprattutto alla diffusione della traduzione in francese delle Mille e Una Notte ad opera dell'orientalista Galland ${ }^{54}$, iniziò ad essere associato ad un'immagine fortemente stereotipata dell'Oriente, nutrita di clichés, miti, di un imperante gusto per l'esotico, il fantastico e il meraviglioso ${ }^{55}$. Quell'«aggregato pittoresco di curiosità», come lo definisce Marie-Noëlle Bourguet ${ }^{56}$, di «oggetti curiosi, rari e preziosi», ispirò viaggiatori, artisti, diplomatici e studiosi di vario genere, e più che essere identificato con uno spazio o un'entità geografica precisi e dai confini delineati (la nozione geo-

(51) Coloro che sbarcavano a Costantinopoli potevano ottenere, direttamente dal Gran Vizir, un lasciapassare o sorta di passaporto che consentisse loro di circolare liberamente in tutto l'Impero.

(52) Generalmente vecchi schiavi cristiani convertiti all'Islam, sorta di rinnegati e reietti dal mondo occidentale, i drogman (o truchement, come spesso venivano definiti in ambito francese nel XVIII secolo) erano personaggi fondamentali, poiché grazie alla propria attività di interpretariato linguistico consentivano e assicuravano la comunicazione tra Oriente e Occidente, altrimenti preclusa. La professione di drogman risaliva in Francia alla creazione dell'École des Jeunes des Langues: nel 1699 Colbert progettò la formazione di giovani interpreti di professione, attribuendo borse di studio a coloro che, una volta terminati gli studi umanistici, venissero inviati presso il Consolato dell'Impero Ottomano per favorire gli interessi commerciali francesi. Pur non essendo specificamente ricompensati a tal proposito, i drogman svolgevano spesso un ruolo delicato nell'ambito della vita diplomatica e politica sia francese, sia orientale, essendo investiti di funzioni di una certa rilevanza. Caso emblematico per tutti quello di Michel Venture de Paradis (1739-1799), che terminerà la propria brillante carriera come "Conseiller général" di Napoleone stesso nel corso della Campagna d'Egitto, dopo aver rivestito le funzioni di segretario-interprete del re e professore di turco all'Ecole des Langues Orientales. Profondamente segnato dalle fatiche della Campagna di Siria, morirà di stenti davanti a S. Giovanni d'Acri nel 1799. Alla fine del XvIII secolo, tuttavia, quella che sembrava una professione prestigiosa cominciò ad essere oscurata da discredito, pregiudizi e riserve: il dubbio cominciò a insinuarsi e il drogman fu da allora considerato un personaggio ambiguo, opportunista, spesso tacciato di imbroglio e spionaggio. Pertanto, accanto alla figura professionale e ufficializzata se ne fece strada una non-ufficiale, comprendente personaggi di basso rango sociale, a volte addirittura reietti, come accennato sopra, assoldati da viaggiatori occidentali in Oriente con la funzione di veri e propri fac-totum. Cfr. SARGA Moussa, op. cit., pp. 13-26.

(53) Sull'interessante tematica del travestimento dei viaggiatori occidentali nei paesi orientali nel XVII, XVIII e XIX secolo cfr.: JoHn RoDEnBECK, Dressing Native, in Janet and Paul Starkey (a cura di), Unfolding the Orient. Travellers in Egypt and the Near East, Ithaca Press, 2001, pp. 65-100.

(54) Orientalista francese (1646-1715), compì numerosi viaggi in Oriente soggiornando a Costantinopoli al seguito dell'ambasciatore di Francia. Lo studio dell'arabo, del persiano e del turco gli consentirono la traduzione del Corano e delle Mille e Una Notte. Mille et Une Nuits. Contes arabes traduits en français par $M$. Galland, Paris, Veuve de Claude Barbin, 1704-1717, 12 voll. in-12 ${ }^{\circ}$.

(55) L'iconografia della letteratura di viaggio nel secolo dei Lumi resta in qualche modo prigioniera dei clichés culturali di cui si nutriva. Spesso le illustrazioni che corredavano i resoconti di viaggiatori non costituivano tanto una testimonianza contemporanea oggettiva, quanto piuttosto una sorta di interpretazione sottomessa parimenti alle correnti artistiche imperanti e ai dettami della politica europea dell'epoca. Tuttavia, benché rivelatrice della concezione occidentale di Oriente, la rappresentazione visiva dei resoconti di viaggio costituisce un prezioso documento. Irini ApOstolou, Les voyageurs français en Orient méditerranéen: leur iconographie au XVIII siècle, conferenza tenuta sul sito www.crlv.org del Centre de Recherche sur la Littérature des Voyages dell'Université de ParisSorbonne, nell'ambito del ciclo Imago Mundi: Lettres et images d'ailleurs.

(56) Marie-Noëlle Bourguet, De la Méditerranée in: MARIE-NoËLle BouRguet (a cura di), L'invention scientifique de la Méditerranée, Paris, Éd. de l'EHESS, 1998, pp. 7-28. 
grafica di Oriente era allora assai vaga ed inconsistente ${ }^{57}$ ), indicava un luogo dell'immaginario collettivo europeo.

Si è in precedenza parlato di termine evocativo, poiché la parola Levante veniva naturalmente associata ad un concetto di rinascita e rigenerazione, secondo una simbologia legata al Sole: il Sole benefico delle origini della cultura, delle radici storiche e linguistiche dell'Antichità, cui si anela ritornare attraverso un viaggio dal profondo valore iniziatico. Si tratta di un viaggio ancestrale verso quella che è considerata la fonte della nostra moderna civiltà, che si cerca di riscoprire e riesumare. In un momento storico di cambiamenti e turbolenze politiche si impone una sorta di nostalgia primitivista, di ritorno ad un glorioso passato. Ma si tratta anche di un viaggio circolare che segna, da un punto di vista cronologico, un continuum, un trait d'union tra passato e presente attraverso la naturale evoluzione e il susseguirsi delle civiltà: da quella egizia antica, a quella ellenistica, sino a giungere a quella latina. Jomard, nel suo Mémoire sur le système métrique des anciens égyptiens ${ }^{58}$, dimostra come i greci abbiano ricevuto dagli egizi le più importanti conoscenze relative non solo ai principi morali e legislativi, ma altresì alle scienze esatte e alle arti liberali. Ne è un esempio il sistema di pesi e misure che i greci hanno tratto da loro, da sempre base fondamentale per il commercio e l'organizzazione della vita sociale. $\mathrm{O}$ ancora, Jollois e Devilliers ${ }^{59}$ sostengono, nel corso della descrizione generale della città di Tebe, che gli antichi egizi avevano un primato incontestabile su altre popolazioni dell'antichità in ambito culturale e che, benché i greci continuassero a vantarsi di aver introdotto in Oriente arti e scienze, ben celando i plagi e furti di cui si erano resi responsabili e protagonisti, in realtà essi avevano un grosso debito di riconoscenza nei confronti dell'antico Egitto.

Perché questo ideale periplo potesse compiersi era necessario tornare nei luoghi un tempo teatro di celebri epopee e riscoprire, attraverso studi e scavi archeologici, le vestigia del passato. L'ordine logico imponeva dunque di ripartire dal glorioso Egitto dei Faraoni. Così come accadde ai savants di Bonaparte, anche i viaggiatori del XVII e XVIII secolo si imbarcarono alla volta dell'Egitto con un bagaglio di saperi e di conoscenze accumulate nei secoli e possedute attraverso i libri in cui erano confluite: le memorie e i resoconti di coloro che avevano viaggiato verso una terra misteriosa ancora tutta da scoprire. L'Oriente in generale cominciava ad apparire come uno spazio familiare. L'esperienza vissuta in prima persona, i resoconti e le nozioni filtrate dalle precedenti tradizioni scientifiche e letterarie si mescolavano determinando una più compiuta percezione del mondo. Nel XVII secolo, ad esempio, abbiamo notizia delle prime missioni archeologiche inviate in Egitto e in Asia Minore, patrocinate e caldeggiate, per quanto riguarda la Francia, dallo stesso ministro Colbert. Lo scopo, oltre all'ispezione scientifica di vestigia del passato, era raccogliere ed acquistare antichi medaglioni, pergamene, preziosi manoscritti arabi e rari oggetti da collezione in genere, attraverso le ingenti somme di denaro che la Corona destinava. Così, il panorama di viaggiatori secenteschi annovera numerose tipologie: ministri, ambasciatori,

(57) Pierre Larousse tentò per primo, nel suo Dictionnaire universel du dix-neuvième siècle, di dare una definizione e delimitazione geografica di Oriente. Jean-Claude Berchet, Le voyage en Orient. Anthologie des voyageurs français dans le levant au XIX siècle, Laffont, 1985, pp. 3-20.

(58) Edme-FrançoIs Jomard, Mémoire sur le système métrique des anciens égyptiens, contenant des recherches sur leurs connoissances géométriques et sur les mesures des autres peuples de l'antiquité, in Description de l'Égypte, Antiquités, Mémoires, vol.

\section{I, 1809 , p. 784.}

(59) Prosper Jollois, Édouard Devilliers, Description générale de Thèbes, in Description de l'Égypte, Antiquités, Descriptions, vol. I, 1809, p. 39. A supporto dell'affermazione del primato egizio su altre civiltà antiche in ambito artisticoscientifico, i due studiosi adducono l'esempio delle cariatidi greche, elementi architettonici destinati a sostenere le colonne dei templi e probabilmente derivati nell'arte ellenica dall'osservazione degli stessi nei templi egizi. 
consoli, commercianti-esploratori, missionari, antiquari in cerca di cammei, statuette o manoscritti. Queste teoriche figure si sono via via incarnate nei celebri nomi di personaggi quali Balthazar de Monconys ${ }^{60}$, Jean de Thévenot ${ }^{61}$, Padre Vansleb e Pietro della Valle ${ }^{62}$.

Con Jean de Thévenot comincia la grande epoca dei viaggiatori ed esploratori di professione. Nel corso del suo lungo viaggio, Thévenot visitò l'Egitto nel 1652 , solo però nelle regioni allora più accessibili, quali il Delta e i dintorni del Cairo. La sua relazione, pur non essendo né ben scritta, né ben organizzata, è apprezzata per la franchezza e la veridicità delle osservazioni e impressioni, che egli si preoccupa di riportare con estrema fedeltà. Il risultato è un affresco assai pittoresco dell'Egitto, caratterizzato da decori, ornamenti e ambientazioni secondo il gusto orientalista allora imperante in Francia. Pur non essendo un archeologo, Thévenot si occupò come molti altri di antichità, in modo particolare di fissare le dimensioni della piramide di Cheope $^{63}$ e di visitare a Saqqara mummie e sarcofagi. Riedita più volte, la sua opera fu molto apprezzata anche nel secolo successivo dai lettori del regno di Luigi XV e seguita da molti viaggiatori, come ad esempio l'olandese Cornelis de Bruin, che la adottò come vero e proprio manuale-guida nel suo viaggio in Egitto ${ }^{64}$.

Padre Vansleb ${ }^{65}$ spicca all'interno di questo gruppo di visitatori non solo per

(60) Balthazar De Monconys, Journal des voyages de M. de Monconys, liutenant criminel au siège présidial de Lyon, où les sçavants trouveront un nombre infini de nouveautez en machines de mathématiques, expériences phisiques, raisonnemens de la belle philosophie, curiositez de chymie et conversations des illustres de ce siècle...etc., Lyon, Boissat, 1665. Dopo aver seguito nel 1618 a Salamanca corsi di filosofia e matematica, Monconys si mise in rotta verso l'Oriente (giungendo ad Alessandria d'Egitto nel 1646) con la speranza di ritrovare le tracce della scienza araba e della filosofia di Zoroastro, da lui studiate precedentemente. Per questo, il suo giornale di viaggio è corredato da innumerevoli digressioni scientifiche e filosofiche. Tuttavia, si possono ritrovare nella sua opera anche utili informazioni e descrizioni della vita quotidiana al Cairo, con le sue guerre di strada, la peste e gli affollati mercati.

(61) JEAN DE THÉVENOT, Relation d'un voyage fait au Levant, contenant diverses particularitez de l'archipel, Costantinople, Terre Sainte, Égypte, des Pyramides, mumies, des déserts d'Arabie, de la Mecque, etc., Paris, 1665. Da non confondersi con lo zio Melchisédech Thévenot, bibliotecario del Re ed eccellente linguista, il quale esercitò una profonda influenza sul nipote, insegnandogli peraltro l'arabo ed incoraggiandolo all'esplorazione. Melchisédech appartiene tuttavia alla categoria di "viaggiatori da camera" e, diversamente dal nipote che ancora giovane perirà sulla strada per la Persia, egli si spegnerà a settantadue anni nel proprio alloggio.

(62) Pietro Della Valle, Viaggi di Pietro Della Valle il Pellegrino, con un minuto ragguaglio di tutte le cose notabili osservate in essi, descritti da lui medesimo in 56 lettere familiari, da diversi luoghi della intrapresa peregrinazione mandate in Napoli all'erudito e fra' più cari, di molti anni suo amico Mario Schipano, divisi in tre parti, cioè la Turchia, la Persia e l'India, le quali avran per aggiunta, se Dio gli darà vita, la quarta parte, che conterrà le figure di molte cose memorabili sparse per tutta l'opera e la loro esplicatione, Roma, 1650. Pietro Della Valle «...da Costantinopoli si diresse in Egitto. Risalendo il Nilo raggiunse Il Cairo e visitò le piramidi. Acquistò sul luogo due mummie che inviò a Roma insieme ad altri oggetti raccolti con la passione del collezionista e dell'antiquario» (Dizionario biografico degli italiani, vol. 37, Roma, Istituto dell'Enciclopedia Italiana $G$. Treccani, 1989, pp. 764-771). La prima parte dei viaggi, cioè quella inerente la Turchia, uscì nel 1650 presso lo stampatore Mascardi. Dopo la morte di Pietro uscirono nel 1658, a cura della moglie e dei figli, il volume relativo alla Persia con dedica ad Alessandro VII, e nel 1663 quello relativo all'India con dedica al Card. Flavio Chigi.

(63) Jean-Marie-Joseph Coutelle, Observations sur les pyramides de Gyzeh et sur les monumens et les constructions qui les environnent, in Description de l'Égypte, Antiquités, Mémoires, vol. II, 1818, p. 39. Coutelle sostiene che Thévenot, così come $i$ viaggiatori moderni Alpin e Bruin e gli antichi Erodoto, Plinio, Strabone e Diodoro Siculo, ha attribuito alla grande piramide un'altezza più o meno uguale alla base.

(64) JeAn-Marie CARRÉ, op. cit., pp. 24-28.

(65) Johann Michael Vansleb, Relazione dello stato presente (1663) dell'Egitto... etc., Parigi, 1671. Johann Michael Vansleb, Nouvelle, Rélation en forme de journal, d'un voyage fait en Egypte. Par le P. Vansleb en 1672 \& 1673, Paris, 1677. Figlio di un pastore luterano di Erfurt ed allievo del grande orientalista Ludolf, Vansleb fu incaricato dal duca Ernest di Sassonia di una spedizione politica e religiosa in Etiopia. Non potendo tuttavia penetrarvi, si fermò in Egitto restandovi per un anno, nel 1664. Di ritorno, sostò a Roma, dove si convertì all'ordine domenicano e fece la conoscenza del vescovo di Montpellier, che successivamente lo presenterà a Colbert nel 1670. Fu proprio il primo resoconto del viaggio in Egitto di Vansleb (redatto in italiano, sua lingua d'adozione), unitamente alla prospettiva di un'alleanza con l'Etiopia a convincere il ministro 
la sua più cospicua presenza entro la Description, ma altresì per essere il primo vero esploratore incaricato ufficialmente dal governo di svolgere una missione al contempo archeologica e religiosa (non abbandonerà infatti l'intento missionario). Il resoconto lasciatoci, pur non brillando a livello linguistico, in quanto redatto in un francese poco più che corretto e chiaro, è apprezzabile per le disquisizioni dotte e ben documentate. I riferimenti dei savants della Description chiariscono ulteriormente l'importanza del contributo apportato da questo impavido esploratore-missionario all'allargamento delle conoscenze relative all'Egitto, benché emerga una certa confusione che, alla fine del XVII secolo, ancora permaneva a livello geografico. Jacotin ${ }^{66}$, nell'esporre le metodologie seguite dagli ingegneri-geografi della campagna per la realizzazione della carta d'Egitto, cita la Nouvelle Relation di Vansleb e le Lettres édifiantes et curieuses del pére Sicard, per quanto riguarda la ricostruzione degli itinerari che dalle rive del Nilo conducevano ai monasteri di Saint-Antoine e Saint-Paul. Di questi ultimi non si avevano appunto notizie se non attraverso le relazioni dei citati Sicard e Vansleb, che li avevano visitati nel corso del loro periplo egiziano. $\mathrm{O}$ ancora, le medesime opere, unitamente alla Relation d'un voyage fait en Égypte en 1730 di Granger, vengono citate da Jacotin per quanto riguarda lo spazio compreso tra la valle dell'Egarement, di Tarfeh, il Nilo e il Mar Rosso, non presente nella carta e ricostruito sulla base delle descrizioni fornite dai tre viaggiatori ${ }^{67}$.

In generale, ai viaggiatori secenteschi la Description reca un doveroso tributo, sottolineando che principalmente a loro si dovevano le conoscenze sull'Egitto giunte sino alla fine del XVII secolo. Tuttavia, i savants non mancano di sottolineare, qua e là, il fatto che molti di essi si plagiano vicendevolmente, indugiando negli errori del passato e perpetrando alcune inesattezze. Per questo, era necessario a volte limitare il credito a loro accordato ${ }^{68}$.

Ma il viaggio inteso come ricerca lascia ben presto spazio a nuove finalità e nuovi obiettivi che si sviluppano e concretizzano parallelamente all'affermazione, alla metà del XVIII secolo, di un modello razionalista nell'ambito della scienza, un modello enciclopedico ed universalista di cultura che mira all'ordinamento e alla classificazione di tutto il sapere umano in categorie distinte ed omogenee: geografia, storia, storia naturale, arte, archeologia, scienza... Il viaggio diviene, per gli spiriti illuminati del Settecento, «enciclopedico nel programma, sistematico nel metodo e geografico nell'obiettivo» ${ }^{69}$. Ma sarà soprattutto con Volney che la cartografia, le statistiche socio-economiche e una certa chiarezza matematica sostituiranno man mano commentari e descrizioni, lasciando così trasparire nuove metodologie di indagine e di razionalizzazione della realtà.

In sostanza, comincia a delinearsi un fine didattico nei resoconti dei viaggiatori, che mettono la propria esperienza personale al servizio della posterità. Già Belon e Postel alla metà del XVI secolo, l'uno per quanto riguarda l'ambito naturalistico, l'altro linguistico, avevano intrapreso il viaggio per soddisfare un bisogno di conoscenza

francese ad affidare una nuova missione al domenicano. Lo scopo era quello di acquistare la maggior quantità possibile di manoscritti, medaglioni e reperti antichi. Vansleb giunse per la seconda volta in Egitto, precisamente al Cairo, nel 1672 e vi rimase per un anno spingendo la propria esplorazione, non senza incorrere in pericoli e difficoltà, più a sud di qualsiasi altro esploratore francese precedente. Sfortunatamente, di ritorno in Francia cadde in disgrazia poiché Colbert, insoddisfatto della sua missione, rifiutò di rimborsargli parte delle spese, lasciandolo così morire in semi-povertà. JEAN-MARIE CARRÉ, op. cit., pp. 29-35.
(66) Pierre Jacotin, Mémoire sur la construction de la carte d'Égypte, in Description de l'Égypte, État moderne, vol. II, $2^{\circ}$ parte, 1822 , p. 49.

(67) Ibid., pp. 99-100. Anche d'Anville sembra aver tratto numerose informazioni dai tre predecessori.

(68) Edme-François Jomard, Mémoire sur le lac de Moeris comparé au lac de Fayoum, in Description de l'Égypte, Antiquités, Mémoires, vol. I, 1809, pp. 9899, e Prosper Jollois, Édouard Devilliers, Description générale de Thèbes, in Description de l'Égypte, Antiquités, Descriptions, vol. I, 1809, p. 93.

(69) Marie-Nö̈lle Bourguet, op. cit., p. 12. 
e per concretizzare, secondo un modello empirico, la propria attività scientifica. Essi inaugurarono così una tendenza alla ricerca del sapere che andò incrementandosi nel XVII e, ancor più, nell' "enciclopedico" XVIII secolo, caratterizzato da un cosmopolitismo ed una effervescenza intellettuali che accompagnavano la vocazione al viaggio e che caratterizzavano la società europea in espansione. Lo spazio visibile e percepibile in Oriente si scopriva per i viaggiatori europei dell'epoca colmo di elementi naturali nuovi e sconosciuti, e la speranza per molti di essi era proprio quella di raccogliere numerose rarità e reperti con i quali arricchire gabinetti privati o collezioni nazionali. In generale, l'apporto dei viaggiatori alle scienze della terra sarà fondamentale anche quando mancheranno loro le condizioni o gli strumenti per osservare, rilevare, studiare. Lo stesso dicasi per quanto attiene la scienza archeologica: infatti, prima della spedizione francese era stato pubblicato in Europa qualche frammento relativo alla scoperta di monumenti antichi in Egitto, in Persia e in India, come ad esempio l'Oedipus Aegyptiacus (1652) di Kircher, l'Antiquité expliquée et représentée en figure (1719. 1724) di Montfaucon o il Recueil d'antiquités égyptiennes, étrusques, grecques et romaines (1752-67) del conte Caylus ${ }^{70}$. Accanto alla moda antiquaria di creare collezioni di oggetti archeologici o naturalistici, cominciano ad apparire opere ragionate che tentino di dare una visione completa e onnicomprensiva del passato. D'altra parte, nella stessa Encyclopédie emerge chiaramente la necessità di ordinare e classificare le nozioni sino allora possedute per superare la generale confusione vigente: «L'histoire de l'Égypte est en général un chaos où la chronologie, la religion, \& la philosophie sont particulièrement remplies d'obscurités \& de confusion. [...] Les connaissances, les coûtumes, les usages, les cérémonies, les idiomes se mêlerent \& se confondirent. [...] On étoit égaré dans un labyrinthe de difficultés réelles» ${ }^{71}$.

Quanto alle precedenti conoscenze geografiche relative all'Egitto, ancora poco consistenti alla fine del XVII secolo, esse si delineano e precisano sempre più chiaramente, colmando le lacune del passato. Se l'Alto Egitto restava scarsamente esplorato e il corso del Nilo pressoché sconosciuto, le ricerche di viaggiatori quali Lucas, Bruce, Sicard, Pococke Norden o Benoît de Maillet contribuiranno a completare il quadro delle conoscenze.

La massiccia ondata esplorativa in Egitto registrata nel corso di tutto il XVIII secolo trae spunto dalla diffusione di opere orientaliste e di compilazioni dotte, come le continue traduzioni del Corano, la circolazione della monumentale Histoire Générale de la religion des Turcs, avec la naissance, la vie et la mort de leur prophète Mabomet o delle Vies de Mahomet, e soprattutto l'Histoire des Arabes del conte Boulainvilliers, che consentiranno di limitare il ritardo accumulato dall'erudizione orientalista di stampo islamico, equiparando le notizie allora possedute sulla Terra dei Faraoni con quelle di altri paesi orientali maggiormente in voga, come India, Cina, Persia o Turchia.

Si procede pertanto attraverso gradi successivi, dalla scoperta vera e propria a cui giunge l'attività esplorativa e che implica il primo contatto e impatto con realtà sconosciute, sino all'investigazione sistematica e scientifica, epurata da sovrastrutture culturali e ombre di ignoranza, che sottende un'analisi approfondita, un approccio

(70) Oltre a nutrire una profonda passione per l'arte e le antichità orientali in genere, Caylus si occupò di letteratura orientalista e fu l'autore nel 1743 di un'opera, i Contes orientaux, generalmente associata alle innumerevoli produzioni settecentesche ispirate alle Milles et Une Nuits di Galland. Per una corretta rilettura del valore e del significato dei Contes di Caylus cfr. Robert Raymonde, Le
Comte de Caylus et l'orient: la littérature aux prises avec le même et l'autre, in «Studies on Voltaire and the Eighteenth Century», n. CLIV, 1976, pp. 1825-1853.

(71) Égyptiens (Philosophie des). Histoire de la Philosophie, in Encyclopédie, ou Dictionnaire Raisonné des Sciences, des Arts et des Métiers, Paris, 1751-72, 17 voll. e riedizioni, textes, vol. 14, p. 70. 
di secondo grado nutrito delle esperienze cronologicamente precedenti. Lo schema esposto riflette in maniera speculare la realtà degli accadimenti e dei successivi progressi registrati nell'ambito della letteratura scientifica e di viaggio: dalle prime relazioni, accolte con favore dal pubblico, di personaggi destinati a lasciare un'impronta indelebile nel panorama culturale, e appartenenti spesso ad un'élite aristocratica per cui il viaggio si configura come un'esperienza privilegiata, quali Thévenot, Vansleb, Lucas, Sicard, Volney ${ }^{72}$, Granger, all'operazione massiccia, coordinata anche a livello militare che coinvolgerà contemporaneamente le più prolifiche menti scelte entro un'intera generazione di intellettuali e studiosi, concretizzatasi storicamente nella spedizione dei savants francesi. Essi porteranno a compimento l'espressione del modello razionalista deputato a combattere le immagini false, i fantasmi e i miti che informavano spesso lo spirito dell'esotismo, sostituendo l'immaginazione con la sfera della sensibilità reale ${ }^{73}$. Si sviluppa così un approccio alla realtà «de nature non plus mentale et synthétique, mais sensorielle et analytique, individuelle e concrète» ${ }^{74}$.

L'elenco dei viaggiatori settecenteschi annoverati dalla Description è vastissimo e contempla i nomi di Volney, Savary, Pococke ${ }^{75}$, Norden $^{76}$, Bruce $^{77}$, Lucas ${ }^{78}$, Gran-

(72) A titolo significativo, citiamo quello di Volney come esempio di viaggio privato, esperienza individuale al di fuori di istituzioni ufficiali, che costituisce una sorta di evoluzione del grand tour, ma le cui finalità e utilità scientifiche vanno a beneficio dell'intera collettività. Egli stesso, in quanto spirito positivista, era un profondo detrattore della curiosità fine a se stessa se non inserita in uno schema di utilità politica o economica, come dimostra chiaramente il resoconto del suo viaggio in Egitto.

(73) Denise Brahimi, Exotisme, science et idéologie, in «Studies on Voltaire and the Eighteenth Century», n. CLI, 1976, p. 369.

(74) Sergio Moravia, Les Idéologues et l'âge des Lumières, in «Studies on Voltaire and the Eighteenth Century», n. CLIV, 1976, p. 1476.

(75) RicHARD POCOCKE, A description of the East and some other countries, London, W. Hawkins, $1743-45,3$ voll.

Con Pococke la passione britannica per le antichità classiche raggiunse l'apogeo. Dopo un lungo viaggiò che lo portò ad attraversare Francia e Italia, lo studioso e teologo inglese visitò tra il 1737 e il 1740 l'Egitto, la Siria, la Terra Santa, Cipro e Costantinopoli. Dopo il suo ritorno a Londra, Pococke divenne uno dei fondatori della Egyptian Society, consacrata alle dotte disquisizioni serali di storia e antichità, che annoverava tra i suoi membri anche Norden, Charles Perry e Montagu. Cfr. Philip Mansel, The Grand Tour in the Ottoman Empire, 1699-1826, in JANET and Paul Starkey (a cura di), op. cit., pp. 41-64.

(76) FredERIK LouIS Norden, Voyage d'Égypte et Nubie: ouvrage enrichi de carte et de figures dessinées par l'auteur, Copenhague, 1755. Giovane ufficiale della Marina Reale Danese, Norden fu protagonista di una missione voluta da Cristiano VI, che aveva inizialmente lo scopo di stabilire rapporti commerciali tra Danimarca, Etiopia e Signore del Madagascar e a cui parteciparono il conte francese d'Esneval con la moglie e altre sedici persone. La spedizione giunse in Egitto nel $1737 \mathrm{e}$ da quel momento Norden si dedicò alla scrupolosa annotazione di tutte le cose interessanti osservate, realizzando peraltro lodevoli disegni e mappe della Valle del Nilo, la cui precisione sarà ammirata anche dal connazionale Niebuhr. Il merito di questo viaggiatore è tanto più evidente in quanto dovette combattere contro costanti pericoli e un precario stato di salute per portare a compimento il proprio lavoro, e sessant'anni prima della spedizione francese fu in grado di fornire descrizioni dettagliate e notizie precise e puntuali di città, villaggi, monumenti. Rientrato in patria incaricò l'amico Tuscher di incidere le 159 tavole che corredano il testo - tratte dai disegni realizzati in loco dallo stesso Norden - e che contribuiscono ad accrescere ulteriormente la qualità e il valore dell'opera del danese. Cfr. Marie-Louise Buhl, ERIK Dal, Torben Colding, The Danish Naval Officer Frederik Ludvig Norden. His travel in Egypt 1737-38 and his Voyage, 1755, Copenhagen, The Royal Danish Academy of Sciences and Letters, 1986.

(77) JAMES BRuce, Travels to discover the source of the Nile in the years 1768, 1769, 1770, 1771, 1772 and 1773, Edinburgh, 1790. Viaggiatore scozzese, nel 1769 penetrò in Etiopia e nel Sudan. Raggiunse le fonti del Nilo azzurro credendo erroneamente di aver scoperto le famose sorgenti del Nilo.

(78) Paul Lucas, Voyage du Sieur Paul Lucas au Levant, Paris, 1704. PAUl LuCAS, Voyage fait en 1714 jusqu'en 1717 par ordre de Louis XIV dans la Turquie, l'Asie, Sourie, Palestine, Haute et Basse Égypte, etc., Paris, 1719. Figlio di un orafo di Rouen, Paul Lucas partì alla volta dell'Oriente per commerciare in pietre preziose. A cominciare dal 1699 riportò numerosi medaglioni che furono acquistati dalla Corona per il gabinetto privato del Re. Nel 1699 si imbarcò per realizzare quel grande viaggio che sarà l'oggetto della sua prima relazione. In esso, Lucas dà conto dell'esplorazione di una parte dell'Alto Egitto, seguendo il corso del Nilo a partire dal Cairo sino a giungere alle cataratte. Rientrato in Francia fu incaricato di una seconda missione, questa volta ufficiale, allo scopo di effettuare ricerche su specie di piante allora sconosciute in Francia e di raccogliere medaglioni e altre curiosità. Da questo momento in avanti i suoi due 
ger $^{79}$, Sicard $^{80}$, Maillet $^{81}$, Niebuhr $^{82}$ e Sonnini ${ }^{83}$, solo per citare i più famosi ${ }^{84}$. Grazie

successivi resoconti di viaggio porteranno l'indicazione par ordre du Roy. L'opera di Lucas è corredata di numerose osservazioni risultanti talvolta da interpretazioni personali fantasiose o desunte dalla compilazione di opere a lui precedenti. Per questo motivo egli è generalmente associato ad esagerazioni, ingenuità o imprecisioni nella descrizione. Ma è possibile che taluni giudizi negativi siano stati forzati dalla mano di detrattori gelosi dell'amatore autodidatta. Anch'egli, come molti altri, incorrerà nel disordine circa l'identificazione di Tebe. A suo merito si rammenta che Lucas può essere definito il primo volgarizzatore, il cui successo renderà popotare non solo la sua opera, ma anche l'Egitto stesso. Cfr. Henry Duranton (a cura di), Voyage du Sieur Paul Lucas dans le Levant, juin 1699 - juillet 1703, Saint-Étienne, Pubblications de l'Université de Saint-Étienne, 1998, pp. 7-15.

(79) Granger, Relation d'un voyage fait en Égypte en l'année 1730, où l'on voit ce qu'il y a de plus remarquables, particulièrement sur l'bistoire naturelle, Paris, 1745. Medico originario di Digione e appassionato di storia naturale, ci ha lasciato una relazione di cui si ammirano la sincerità e l'impressione di veridicità.

(80) Claude Sicard, Nouveaux mémoires des missions de la Compagnie de Jésus dans le Levant, Paris, 1727. Superiore della missione gesuita al Cairo, Sicard fu dapprima professore a Lione e successivamente missionario in Siria. Si stabilì al Cairo dal 1712 e vi morì di peste nel 1726. Dotato di spirito scientifico e di curiosità critica, era anche un eccellente linguista e conoscitore della lingua araba. A differenza di Vansleb egli non fu incaricato ufficialmente dal governo di viaggiare in Oriente, ma fu spinto dal proselitismo del missionario unito ad uno spiccato interesse archeologico. Sfortunatamente, le sue memorie manoscritte relative all'Egitto non furono pubblicate e andarono perdute dopo la sua morte. Oltre ai Nouveaux mémoires da lui composti successivamente e alle Lettres édifiantes et curiueses de la Société de Jésus, ci è rimasta di Sicard una carta manoscritta antica (Carte de l'Égypte ancienne et moderne, Paris, 1757-58) di rara precisione ed esattezza, sicuramente consultata anche dal celebre geografo d'Anville. Sicard ha inoltre il merito di aver descritto, circa una ventina d'anni prima di Pococke, il portico del tempio di Hermopolis e di essersi spinto sino ad Assouan risalendo il corso del Nilo. Cfr. JeAn-MARIE CARRE, op. cit., pp. 49-53.

(81) Benoît de Maillet, Description de l'Égypte, contenant plusieurs remarques curieuses sur la Geographie ancienne et moderne de ce Païs, sur ses Monumens anciens, sur les Moeurs, les Coûtumes E la Religion des Habitans, sur le Gouvernement \& le Commerce, sur les Animaux, les Arbres, les Plantes, Ec. Composée sur les Mémoires de Monsieur de Maillet, Ancien Consul de France au Caire, par M. l'Abbé Le Mascrier. Ouvrage enrichi de Cartes E de Figures, Paris, 1735. Gentiluomo della Lorena nato nel 1656, spirito vivo, intelligente e aperto ad ogni curiosità, Benoît de Maillet è, prima di Volney, il viaggiatore che ha maggiormente contribuito ad estendere le conoscenze settecentesche dell'Egitto. Fu inviato al Cairo nel 1692 e qui trascorse sedici anni. Alla fine della carriera consolare, ritiratosi a Marsiglia, compose delle memorie relative alla sua esperienza in Egitto, acquistate da un editore e successivamente arrangiate e pubblicate, mentre egli era ancora in vita, dall'abate Le Mascrier. La sua Description, prima opera d'insieme sull'Egitto, non è un resoconto di viaggio, ma uno studio sintetico e didattico che si pone l'obiettivo di riassumere tutte le conoscenze allora circolanti su tale soggetto. Questo chiarisce gli intenti dell'autore: istruire piuttosto che divertire, e in tal senso egli si porrà in aperto contrasto con $i$ viaggiatori a lui precedenti o contemporanei. Benché permangano nell'opera diverse imprecisioni geografiche, Maillet ebbe il merito, dopo Villamont e Thévenot, di aver correttamente identificato la città di Tebe. Presumibilmente, invece, non visitò mai l'Alto Egitto. Cfr. JeAn-Marie Carré, op. cit., pp. 56-63.

(82) Carsten Niebuhr, Beschreibung von Arabien: aus eigenen Beobachten und im Lande selbst gesammelten Nachrichten, Kopenhagen, Nicolaus Möller, 1772. Niebuhr, allora luogotenente dell'esercito danese, fu l'unico sopravvissuto alla spedizione in Arabia del 1761-67, voluta dal re di Danimarca, di cui era stato nominato tesoriere. Topografo di formazione, egli era stato inoltre incaricato di occuparsi di misurazioni e dopo la morte dei propri compagni di viaggio (Frederik Christian von Haven, filologo - Christian Carl Kramer, medico e fisico - Georg Wilhelm Baurenfeind, disegnatore - Peter Forsskål, botanico) continuò l'esplorazione dell'India, della Persia e del Levante. È considerato a buon diritto uno dei più grandi viaggiatori del XVIII secolo per la qualità dei suoi lavori, oltre che per l'oggettività e scientificità delle argomentazioni, attraverso cui si proponeva di cancellare i clichés allora imperanti.

(83) Sonnini de Manoncourt, Voyage dans la Haute et la Basse Égypte fait par ordre de l'ancien gouvernement et contenant des observations de tous genres, Paris, 1799. Spirito vivo, positivista, appassionato di esplorazione e scienza, quest'ufficiale e ingegnere della Marina s'imbarcò nel 1777 alla volta del Vicino Oriente. Di ritorno in Francia dopo tre anni, nel 1780, invece di redigere immediatamente il suo resoconto di viaggio, si dedicò alla stesura di numerosi articoli di ornitologia. Il suo Voyage è considerato opera più completa rispetto a quella di Volney e Savary: altrettanto preciso rispetto al primo, ma meno elegante rispetto al secondo, Sonnini è tuttavia caratterizzato da un campo di osservazione di gran lunga più vasto rispetto ai due predecessori, comprendente botanica, mineralogia, zoologia, medicina, ornitologia, ittiologia e altro ancora.

(84) Non dimentichiamo inoltre Georg Zoëga, John Greaves, Thomas Shaw, Cornelis de Bruin. GEORG ZOËGA, De origine et usu obeliscorum, Roma, 1797. Questo connazionale di Norden archeologo, numismatico ed esperto di copto otterrà importanti risultati riguardo all'osservazione di obelischi e ge- 
alla loro audacia e temerarietà le nozioni geografiche ed etnografiche sull'Egitto registreranno notevoli progressi. La Description si pronuncia nei loro confronti usando toni a volte elogiativi, («hommes aussi recommandables» ${ }^{85}$ ), altre volte più critici, come quando, a proposito di un'erronea congettura elaborata da Bruce sui blocchi granitici di Lambâgeh, Rozière afferma:

Cette partie de l'ouvrage, la seule que je me permette de juger, est remplie d'assertions aussi peu fondées. [...] Je passe sous silence ses méprises en minéralogie. La manière dont il s'exprime prouve suffisamment qu'il étoit peu versé dans cette partie de l'histoire naturelle; mais j'ai regardé comme indispensable de relever les autres méprises... ${ }^{86}$

\section{o ancora:}

Nous avons les preuves de ces erreurs; l'observation nous a montré constamment que les Égyptiens n'ont été chercher au loin que ce qu'il leur étoit impossible de trouver près d'eux ${ }^{87}$.

Tuttavia, non va mai dimenticato che le inesattezze in cui alcuni viaggiatori sono a più riprese incappati derivavano spesso dalla mancanza di idonee condizioni di lavoro ed osservazione. All'interno della descrizione generale di Tebe, ad esempio, Jollois e Devilliers affermano con forza:

Pendant notre séjour dans la haute Égypte, nous avions, pour en étudier les monumens, des facilités qui ont manqués aux voyageurs qui nous ont précédés: isolés et sans défense, ils étoient en butte à l'avarice et à la jalousie d'une population ignorante, fanatique et cruelle; leur vie étoit continuellement menacée; ils ne pouvoient faire un pas sans être rançonnés: ce n'est qu'à la hâte et en courant qu'ils ont pu voir les monumens ${ }^{88}$.

Parimenti, questo spiegherebbe le lacune e imprecisioni relative alla descrizione di monumenti, sculture e bassorilievi e l'impossibilità a consacrarsi a osservazioni lente e minuziose:

Les voyageurs qui avoient parcouru l'Égypte avant nous, n'avoient pu se consacrer à des observations lentes et pénibles. La plupart n'ont point pénétré dans l'intérieur des édifices; des recherches aussi rapides, et souvent troublées par le sentiment du danger personnel, ne pouvoient donner que des résultats très-imparfaits ${ }^{89}$.

Tra coloro che hanno preceduto la campagna napoleonica Pococke e Norden sono gli unici ad avere il merito di aver fornito i maggiori particolari sull'architettura degli antichi monumenti egizi ${ }^{90}$. I savants al contrario hanno potuto operare con meno inquietudine e pericolo grazie alla protezione militare dell'esercito, ben

roglifici e la sua opera inaugurerà una nuova epoca. CORNELIS DE BRUIN, Voyage au Levant, c'est-à-dire dans les principaux endroits de l'Asie Mineure, dans les isles de Chio, Rhodes, Chypre etc. de même que dans les plus considérables villes d'Égypte, Syrie E Terre Sainte..., Rouen, Charles Ferrand, 1725 , ThOmas SHaw, Voyages dans plusieurs provinces de la Barbarie et du Levant, contenant des observations géographiques, physiques, philologiques..., trad. de l'anglais, La Haye, 1743, John Greaves, Pyramidographie, London, 1646.

(85) Étienne Geoffroy-Saint-Hilaire, Description des crocodiles d'Égypte, in Description de l'Égypte, Histoire Naturelle, vol. I, 1809, p. 245.
(86) Michel RozIÈRE, Description minéralogique de la Vallée de Qoçeyr, in Description de l'Égypte, Histoire Naturelle, vol. II, 1813, pp. 95-96.

(87) Ibid.

(88) Prosper Jollois, Édouard Devilliers, Description générale de Thèbes, in Description de l'Égypte, Antiquités, Descriptions, vol. I, 1809, pp. 395-396.

(89) JOSEPH FOURIER, Premier mémoire sur les monumens astronomiques de l'Égypte, in Description de l'Égypte, Antiquités, Mémoires, vol. II,1809, p. 81.

(90) Prosper Jollois, Édouard Devilliers, op. cit., p. 263 , nota I. 
comprendendo la posizione privilegiata rispetto ad altri studiosi europei e la grande e irripetibile occasione loro offerta.

Una menzione particolare è necessaria nei confronti del celebre geografo J. B. Bourguignon d'Anville ${ }^{91}$ che, pur non appartenendo alla categoria dei viaggiatori, attraverso il prodigioso lavoro svolto nel proprio gabinetto privato, si rivelerà punto di riferimento fondamentale per gli ingegneri della spedizione. La carta dell'Egitto, da lui realizzata nel 1765 affiancando la moderna geografia allo studio di quella antica per ottenere migliori risultati, fu seguita dalla spedizione e servì a guidare le operazioni militari. La sua utilità spinse infatti Jomard ad inserirla all'interno della Description:

Il est vrai que les cartes de d'Anville et ses mémoires géographiques sur l'Égypte jouissent, et à juste titre, d'une haute réputation; il n'est pas un voyageur, pas un érudit, à qui ces travaux n'aient été de plus grand secours. Sa carte a été un guide presque toujours sûr pour l'expédition Française, elle a servi au général, à l'astronome, à l'ingénieur, à l'artiste, à l'antiquaire: aussi nous avons toujours payé un tribut d'admiration au mérite de cette production, que l'auteur affectionnoit d'une manière particulière ${ }^{92}$.

Appariva davvero sorprendente l'abilità con cui d'Anville era stato in grado, dal suo gabinetto privato, di distinguere contraddizioni ed inesattezze riportate dalle memorie dei vari viaggiatori in Egitto. Nei suoi confronti i savants hanno usato a più riprese espressioni quali «habile homme» ${ }^{93}$ «...qui se trompe rarement...» ${ }^{94}$, dimostrando come egli fosse capace di giungere spesso alla verità attraverso uno spirito pieno di sagacia e la formulazione di giudizi corretti. Accorto e moderato, serbava invece il silenzio laddove non aveva a disposizione dati sicuri. Significative a tal proposito le parole di Jacotin:

C'est à lui qu'il faut toujours recourir, lorsqu'on est dans le cas de donner des cartes de pays où les voyageurs modernes n'auront pu encore pénétrer ni faire observations. On ne peut qu'admirer la sagacité avec laquelle ce savant géographe a pu, au fond de son cabinet, assigner à chaque lieu sa position respective ${ }^{95}$.

Prima di prendere in considerazione la figura che più di ogni altra nel panorama settecentesco ha lasciato un segno distintivo, anticipando lo spirito della Description

(91) Jean Baptiste Bourguignon D’Anville, Mémoires sur l'Égypte ancienne et moderne suivis d'une description du Golfe Arabique ou de la Mer Rouge, Paris, Imprimerie Royale, 1766.

(92) EDME-FranÇOIS JomARD, Explication des planches d'antiquités. Planches grand format, relatives à la géographie comparée, in Description de l'Égypte, Antiquités, Mémoires, vol. II, 1818, p. 149. Benché le carte geografiche di d'Anville e le sue memorie sull'Egitto godessero a buon diritto di un'alta reputazione, e per quanto quest'illustre geografo fosse riuscito ad includere con grande sagacia ed esattezza nella sua carta i principali siti egiziani, quelli riportati risultavano essere in numero insufficiente o riprodotti in scala troppo piccola. Si rese così necessario il lavoro di tutti gli ingegneri geografi della spedizione incaricati di effettuare rilievi e operazioni trigonometriche per la realizzazione di una carta più completa dell'Egitto. È necessario ammettere, infatti, che le successive osservazioni geografiche e le operazioni di ingegneria avevano modificato alcuni dati di fatto. In nota 2 p. 148 , Jomard precisa che la carta generale dell'Egitto, redatta dal colonnello Jacotin e dalla sua squadra di ingegneri-geografi, fu presentata alla Commission d'Egypte il 22 agosto 1814, mentre le restanti carte furono sottoposte all'Institut de France qualche anno più tardi.

(93) EDME-FrançoIs JOMARD, Mémoire sur le système métrique des anciens égyptiens, contenant des recherches sur leurs connoissances géométriques et sur les mesures des autres peuples de l'antiquité, in Description de l'Égypte, Antiquités, Mémoires, vol. I, 1809, p. 633.

(94) Étienne Geoffroy-Saint-Hilaire, Description des crocodiles d'Égypte, in Description de l'Égypte, Histoire Naturelle, vol. I, 1809, p. 252.

(95) PierRe Jacotin, Mémoire sur la construction de la carte d'Égypte, in Description de l'Égypte, État moderne, vol. II, $2^{\mathrm{a}}$ parte, 1822 , p. 103. 
e costituendo a buon diritto un trait d'union con essa, l'idéologue francese Volney, è necessario menzionare un altro suo connazionale, anch'egli protagonista negli stessi anni di un viaggio in Egitto, ma le cui conclusioni saranno tuttavia profondamente dissimili. Claude-Étienne Savary ${ }^{96}$ è un viaggiatore colto, documentato, brillante e spontaneo, dotato delle qualità dello scrittore elegante e vivace al tempo stesso. La sua opera appartiene alla tradizione del racconto sotto forma di raccolta epistolare, che unisce l'aneddoto alla delineazione di scene pittoresche e al gusto per la digressione narrativa. Il vantaggio che una tipologia del genere potesse presentare era costituito dal fatto che il ritmo del viaggio, delle sue emozioni e sensazioni poteva essere seguito naturalmente, senza altre costrizioni di tipo narrativo. Nonostante i detrattori, primo fra tutti Volney, l'abbiano accusato a più riprese di essersi abbandonato in modo troppo disinvolto a visioni incantate e romantiche o ad elementi piuttosto favolosi che risentivano dell'immaginario settecentesco legato all'Oriente, proprio i savants contribuiranno a mitigare la durezza del giudizio formulato nei suoi confronti. Così Chabrol affermerà con forza:

Nous emprunterons à ce sujet quelques réflexions à l'auteur des Lettres sur l'Égypte, qu'on a si amèrement et quelquefois si injustement critiqué: elles peignent parfaitement les nuances qui existent entre les usages des peuples de l'Orient et ceux de l'Occident, à l'égard de la vieillesse ${ }^{97}$.

Giungiamo finalmente a colui che incarna in modo più significativo la visione razionalista ed enciclopedica del viaggio. Volney ${ }^{98}$, uno dei più famosi viaggiatori dell'epoca illuministica, fu inviato in missione segreta in Oriente per analizzare la situazione politica e morale dell'Impero Ottomano, e in modo particolare dell'Egitto, paese messo in ginocchio dal dispotismo, allo scopo di valutare la fattibilità di un progetto di invasione francese. Egli è un filosofo caratterizzato da un desiderio di oggettività scientifica in nome della Ragione e dello spirito degli idéologues ${ }^{99}$ a cui

(96) Claude ETIEnNe Savary, Lettres sur l'Égypte où l'on offre le parallèle des moeurs anciennes et modernes des babitans, où l'on décrit l'état, le commerce, l'agricolture..., Paris, 1785-86. Nato nel 1750, Savary fece solidi studi al Collège de Rennes e partì nel 1776 per l'Egitto, dove si fermò tre anni. Rientrato in Francia pubblicò una traduzione del Corano, una Vie de Mahomet e una Morale de Mabomet. Soprattutto, si dedicò alla stesura delle sue Lettres, opera in aperto contrasto con quella di Volney. Queste Lettres, dopo aver goduto di un grande successo, furono investite da pesanti critiche da parte dei detrattori. Cfr. JeAn-Marie Carré, op. cit., pp. 80-90.

(97) GIlbert Chabrol de Volvic, Essai sur les moeurs des habitans modernes de l'Egypte, in Description de l'Égypte, État moderne, vol. II, $2^{\mathrm{a}}$ parte, 1822, pp. 445-446.

(98) Constantin-François Volney, Voyage en Syrie et en Égypte pendant les années 1783, 1784 et 1785, Paris, Volland, 1787, 2 voll. Nato nel 1757 e figlio di un avvocato rimasto vedovo dopo due anni dalla sua nascita, Volney si chiamava in realtà Costantin François Chasseboeuf, nome che muterà nel più famoso pseudonimo, si dice in onore dell'ammirato Voltaire, da cui trasse la prima sillaba del nome e l'ultima di Ferney, luogo in cui viveva il filosofo. Cfr. JeAn VerCOUTtER, Le rêve orientale de Bonaparte, in AA. Vv., Mémoires d'Égypte: hommage de l'Europe à Champollion, Strasbourg, La Nuée Bleue, 1990, p. 23. Di carattere introverso e salute debole, votato agli studi e all'osservazione, approfondì in gioventù lo studio della medicina, del greco e dell'arabo, interessandosi anche di climatologia, geologia, agronomia ed economia rurale. Nozioni che egli mise poi a frutto durante il suo viaggio in Egitto e Siria. Volney si imbarcò nel 1782 per il Vicino Oriente, dove rimase per circa tre anni sino al 1785, grazie ad un improvviso ed inaspettato lascito. Nel corso del viaggio in Egitto, la prudenza lo spinse a muoversi in carovane, il che gli permise di visitare solo le aree allora genericamente battute dai viaggiatori: Alessandria, il Cairo, Damietta e Suez. Sulla poliedrica figura di Volney si veda in generale JEAN GAULMIER, L'idéologue Volnev: 17571820. Contribution à l'bistoire de l'orientalisme en France, Genève-Paris, Slatkin, 1980.

(99) Sui philosophes e gli idéologues si vedano: LIONELLO SOZZI, Un concorso dell'Institut su "L'indépendance de l'bomme de lettres", in DanIELA GALLINGANI (a cura di), op. cit. e Il Principe e il filosofo. Intellettuali e potere in Francia dai Philosophes all'Affaire Dreyfus: saggi, Napoli, Guida, 1988. Sergio Moravia, Les Idéologues et l'áge des 
appartiene. In lui si fondono dunque la registrazione attenta e meticolosa di ogni dato secondo una griglia di lettura preordinata, e la profonda analisi e riflessione che ne scaturiscono successivamente. Il risultato, dopo tre anni di peregrinazioni, sarà il Voyage en Syrie et en Égypte, opera che ebbe grande fortuna e le cui considerazioni faranno scuola. Attraverso essa Volney abbraccia un tipo di relazione scientifica dai toni rigorosi, che esclude divagazioni ed elementi romanzeschi per trasformarsi in un repertorio di informazioni e analisi tematiche.

Volney interrompe così bruscamente quell'idealizzazione romantica caratterizzata da toni trasognati e idilliaci e comune a tanti suoi contemporanei, primo fra tutti Savary, per spingersi verso una sorta di inchiesta sociologica. Convinto dell'appartenenza del genere "viaggio" alla categoria della storia, piuttosto che a quella del romanzo, egli abbraccia l'oggettività e l'autenticità scientifiche epurate dalle strutture immaginative che normalmente permeano lo spirito dell'osservatore. Uomo austero e riservato, poco sollecito all'immaginazione, Volney intraprende il viaggio per soddisfare la propria curiosità intellettuale. Le ragioni addotte non si esauriscono unicamente nell'interesse culturale, ma investono altresì il campo politico-sociale, che lo spinge a registrare meticolosamente i tratti caratteristici e lo stato fisico e politico dell'Impero Ottomano, non senza un risvolto ideologico di stampo imperialista ${ }^{100}$. Diversamente dalla maggior parte dei precedenti viaggiatori, per lo più preoccupati di studiare il bagaglio di antichità di un paese, Volney abbandona, secondo Nicole Hafid-Martin, il progetto di comparatismo storico per dedicarsi ad una sintesi contemporanea $^{101}$ che ponga l'accento sui moderni stati egiziano e siriano. Ed è precisamente in tal senso che la sua esperienza è emblematica della mutata concezione scientifica del viaggio nell'epoca illuministica e costituisce un trait d'union fondamentale con lo spirito della Description. Peraltro l'opera di Volney eserciterà un profondo influsso e finirà per costituire la fonte primaria a cui Napoleone stesso attingerà per l'organizzazione della campagna d'Egitto. Come confermano le parole di Fourier:

Un écrivain justement célèbre, en publiant les résultats de ses voyages en Égypte et en Syrie, avoit déjà enrichi la littérature Française d'une description éloquente et exacte des mœurs et du gouvernement de ces contrées. On sait que la vérité de ses observations est confirmée par les recherches qui ont été entreprises pendant le cours de l'expédition ${ }^{102}$.

Il discorso su Volney ci porta direttamente alla Description de l'Égypte, che possiamo a giusto titolo definire figlia dello spirito scientifico ed enciclopedico settecentesco, così come del bisogno, tanto caro alle «idee umanistiche e al culto del sapere degli studiosi francesi della fine del XVIII secolo ${ }^{103} \gg$, di percezione allargata del mondo e delle sue categorie cognitive. La luce diviene così metafora della conoscenza, e la storia un'evoluzione della conoscenza stessa. L'iniziativa storica è lasciata a uomini

Lumières, in «Studies on Voltaire and the Eighteenth Century», n. CLIV, 1976, pp. 1466-1467 e Il pensiero degli "Idéologues", scienza e filosofia in Francia, 1780-1815, Firenze, 1974.

(100) Partendo dal presupposto della superiorità ideologica occidentale e della necessità di un intervento dei Lumi europei in Oriente, Volney preconizza che la missione civilizzatrice in Egitto possa anche assumere la forma di una conquista militare. Egli è convinto che le province ottomane mal sopportino il giogo turco, ma, persuaso dalla storia recente, ritiene che una rivoluzione non debba essere condotta dall'interno, poiché è fondamentale un intervento esterno che guidi il popolo oppresso alla liberazione. Su questo punto si legga in generale il capitolo «L'enquête socio-politique de Volney dans les provinces turques (Égypte et Syrie)», in NicolE Hafid-MarTin, op. cit., pp. 101-21.

(101) Cfr. ibidem, pp. 58-59.

(102) Description de l'Égypte, Préface et explication des planches, 1809, Préface Historique, p. LXXXIV.

(103) Franco SERINO, 1798-1801, Napoleone e l'Egitto. Il bicentenario della riscoperta, Torino, Ananke, 1999, p. 54. 
illuminati che assumano il compito di comunicare e diffondere verità assolute per un generale miglioramento della società ${ }^{104}$. Lo stesso Volney affermava: «Ce n'est qu'en rassemblant ce qu l'on a vu par soi-même et ce que d'autres ont observé que l'on peut acquérir quelques idées générales» ${ }^{105}$. Uno dei temi maggiormente sfruttati dalla retorica illuminista, e applicato anche a fini di propaganda politica, è, come afferma giustamente Lionello Sozzi, quello della «funzione didattica dell'uomo di lettere, "précepteur des humains" (così dice un testo), la cui penna istruisce ed "éclaire", il cui compito cioè è quello di diffondere i lumi nel mondo, di propagare "les saintes vérités", di “réculer les barrières" che ostacolano l'espandersi del sapere» ${ }^{106}$. Viaggio e scienza sono così associati in una nuova metafora, per significare che l'attività esplorativa porta alla scoperta, in altre parole, alla conoscenza. Personalità come quella di Volney, uomo di pensiero e d'azione e figura più compiuta di philosophe, mettono dunque il proprio talento di scrittore al servizio del rinnovamento sociale e del militantismo politico. La grande innovazione introdotta da Bonaparte e il carattere unico e rivoluzionario che possiamo a buon diritto riconoscere alla spedizione d'Egitto e conseguentemente alla Description è rappresentato dall'aver dato all'impresa la forma di missione dotta, organizzata sotto la tutela dello Stato e il patrocinio delle istituzioni scientifiche, e condotta sul terreno da un gruppo di studiosi reclutati a tale scopo, che lavorano protetti dall'esercito e talvolta in collaborazione con esso. Il legame tra scienza e guerra si precisa e si complica entro un generale disegno che subordina il sapere alla conquista, mettendolo al servizio della diplomazia. Ai fini dichiaratamente politici, l'attività scientifica diviene così elemento indispensabile sulla scena internazionale per suggellare la gloria dei Principi o la grandezza degli Stati ${ }^{107}$.

Ma questo non è il solo tratto distintivo e rivoluzionario che contribuisce ad individuare e delineare una profonda separazione tra la Description e le precedenti opere della letteratura di viaggio. Tutto un contesto ideologico caratteristico della fine del XVIII secolo concorrerà ben presto a colorire il didattismo positivista insito nella filosofia dei Lumi di istanze imperialiste e colonialiste. Là dove i precedenti viaggiatori avevano proiettato in Egitto l'immagine legata al mito di un'antichissima civiltà, il quadro entro cui nasce la spedizione e, di conseguenza, la Description, è quello delle speculazioni filosofiche degli idéologues, miranti a dimostrare come le nefaste conseguenze della tirannia politica vigente nell'Impero Ottomano potessero essere risollevate da un intervento europeo ${ }^{108}$. Non a caso, la propaganda francese cercherà di diffondere un'immagine idealizzata dell'operazione napoleonica in terra d'Egitto, che sottolinei con forza la volontà di creare una relazione pacifica tra Oriente ed Occidente, malgrado la presenza dell'esercito. Questo si poneva naturalmente in linea con gli obiettivi che la spedizione, almeno ufficialmente, si era proposta, riassumibili nella volontà e necessità di esportare al di fuori dei confini francesi i fermenti

(104) Michel Delon, Les Lumières: travail d'une métaphore, in «Studies on Voltaire and the Eighteenth Century», n. CLII, 1976, p. 530.

(105) Constantin-Francois Volney, Voyage en Syrie et en Égypte pendant les années 1783, 1784 et 1785, Paris, Volland, 1787, 2 voll. Edizione consultata, Paris, Volland-Desenne, 1789, p. 12.

(106) Cfr. Lionello Sozzi, op. cit., p. 137. La teoria degli idéologues trovava immediata applicazione anche in ambito politico nella convinzione che la diffusione dei Lumi consentisse a lungo andare «a tutta la società di partecipare alla gestione di se stessa, di beneficiare di una vera uguaglianza e di una vera libertà», posizione questa che consentiva loro di fondere tendenze elitiste con una generale, ma contrastante, tendenza democratica. Cfr. Yves BÉNOT, Il gruppo della "Décade Philosophique": un tentativo di resistenza intellettuale (1799-1803), in Daniela Gallingani (a cura di), op. cit., p. 99.

(107) Marie-Noëlle Bourguet, Des savants à la conquête de l'Égypte? Science, voyage et politique au temps de l'expédition française, in PATRICE BRET (a cura di), L'expédition d'Égypte: une entreprise des lumières, 1798-1801, Actes du colloque international organisé par l'Académie des Inscriptions et Belles Lettres..., Paris, Tec \& Doc, 1999, pp. 21-36.

(108) Si veda a tal proposito ANDRÉ RAYMOND, Les Égyptiens et les Lumières pendant l'expédition française, in PATRICE BRET (a cura di), L'expédition d'Égypte, cit., 1999, pp. 103-317. 
rivoluzionari e i principi di civismo, di uguaglianza politico-sociale e di lotta contro le limitazioni alla libertà e ai principali diritti dell'uomo. In sostanza, contro ogni forma di dispotismo, brutalità, fanatismo ed ignoranza ${ }^{109}$. Così, sostiene con forza Fourier:

Elle [l'Égypte] gémit aujourd'hui sous l'autorité la plus arbitraire et la plus imprévoyante qui ait encore existé sur la terre; [...] Ils oppriment toute industrie, abandonnent ou détruisent les canaux et les monumens publics; L'homme est condamné, dans les campagnes, à un travail ingrat, dont les fruits ne doivent point lui appartenir; par-tout il est en proie à l'injustice, à l'opprobre, à la famine, aux maladies contagieuses ${ }^{110}$.

\section{E ancora:}

L'autorité du souverain ne peut ni assurer l'envoi de tributs, ni protéger les peuples, ni garantir l'exécution des traités faits avec les puissances alliées. Ce sont ces dernières circonstances qui déterminèrent l'expédition mémorable des Français: mais le Héros qui la dirigea, ne bornoit point ses vues à punir les oppresseurs de notre commerce; il donna au projet de cette conquête une élévation et une grandeur nouvelles, et lui imprima le caractère de son propre génie. Il apprécia l'influence que cet événement devoit avoir sur les relations de l'Europe avec l'Orient et l'intérieur de l'Afrique, sur la navigation de la Méditerranée et le sort de l'Asie. Il s'étoit proposé d'abolir la tyrannie des Mamelouks, d'étendre les irrigations et la culture, d'ouvrir une communication constante entre la Méditerranée et le Golfe Arabique, des former des établissemens de commerce, d'offrir à l'Orient l'utile exemple de l'industrie Européenne, enfin de rendre la condition des habitans plus douce et de leur procurer tous les avantages d'une civilisation perfectionnée ${ }^{111}$.

In tal senso, la Description era stata caricata del ruolo di manifesto programmatico dell'ideologia napoleonica, che, dietro la duplice giustificazione fornita dal progetto di importare cultura esportando civiltà, nascondeva ben altre mire politicomilitari ed economiche. In realtà, è emerso chiaramente come la spedizione francese abbia anche dato vita ad una serie di approcci sistematici nei confronti dell'Oriente in cui il denominatore comune fu, oltre all'esplorazione geografica e scientifica, lo sfruttamento politico-economico, celato dietro al decantato potere redentore dei Lumi e alla missione civilizzatrice della cultura europea:

Tels sont les avantages naturels de l'Égypte, qu'ils n'ont pu être entièrement anéantis par la longue influence d'une administration vicieuse. On y jouit encore aujourd'hui des richesses de l'agriculture, de celles de l'industrie et du commerce. Ainsi, l'Égypte a conservé tout les élémens de son ancienne grandeur; et ces germe précieux d'une prospérité nouvelle se développeroient rapidement, s'ils étoient fécondés par le génie de l'Europe et les bienfaits d'un gouvernement sage et puissant ${ }^{12}$.

Sempre nella Préface Historique, Fourier, cercando una giustificazione all'espansionismo politico francese, affermerà paradossalmente la necessità di un intervento in Egitto per ristabilire il rispetto delle negoziazioni commerciali e per porre fine agli innumerevoli soprusi e vessazioni patiti dai suoi connazionali:

Les négocians étrangers ne furent point garantis de cette oppression; les Français surtout essuyèrent des vexations et des outrages qui demeurèrent long-temps impunis. [...] Les extor-

(109) Le conquiste militari francesi si ritenevano funzionali alla penetrazione dei Lumi e alla propagazione dell'istruzione nei paesi "liberati" dai transalpini, ma che di fatto rischiavano di essere sottoposti ad un nuovo giogo.
(110) Description de l'Égypte, Préface et explication des planches, 1809, Préface Historique, pp. V-VI.

(111) Ibid., pp. V-VI.

(112) Ibid., pp. XXXIJ. 
sions et les insultes se renouvelèrent et entraînèrent la ruine de nos maisons de commerce. On ne pouvoit point les abandoner, sans livrer à des nations rivales des avantages que l'on devoit aux plus anciens traités, et sans donner un example de foiblesse qui seroit devenu fatal à tous les établissemens Français. [...] Ces circonstances ont appelé les Français en Égypte, et ce pays est devenu le théâtre d'un des plus grands événemens de l'histoire moderne. Aux motifs que l'on vient de rappeler, se joignoient la considération des avantages que promettoit un établissement fixe dans le Levant ... ${ }^{113}$

Uno degli elementi probanti di questa tendenza imperialista fu il tentativo di portare a termine, già durante l'occupazione francese dell'Egitto, quel progetto che avrebbe poi trovato piena realizzazione nell'apertura del Canale di Suez. L'idea di un canale che congiungesse Alessandria con Suez fu elaborata infatti dall'ingegnere Lepère, membro della spedizione di Bonaparte, piano al quale successivamente l'ingegnere Linant de Bellefonds, al Servizio di Méhmet Ali, aveva sostituito quello di un tracciato diretto. Di quest'ultimo progetto doveva certamente essere a conoscenza Ferdinand de Lesseps, console francese ad Alessandria negli anni 1834-1835 e 1836-1838 a cui si deve la realizzazione del Canale. Il Canale di Suez, ideato per congiungere e mettere in collegamento il Mar Mediterraneo e il Mar Rosso, fu inaugurato il 17 novembre 1869 dall'imperatrice Eugenia, e costituisce non solo, come sostiene Edward Said, «la conclusione logica del pensiero orientalista» e il culmine dell'ideologia bonapartista, ma altresì «lo sforzo occidentale di invadere e dominare l'Oriente» ${ }^{14}$. A partire da quel momento, l'Egitto cessa di essere mediterraneo per divenire internazionale ${ }^{115}$. Le barriere cognitive sono ormai definitivamente abbattute e i segreti custoditi dall'antica terra dei faraoni destinati ad essere progressivamente svelati dalla civiltà occidentale.

Punto di svolta nei rapporti tra Vicino Oriente ed Europa, l'invasione napoleonica dell'Egitto ha finito per costituire un modello di appropriazione scientifica di una cultura ai danni di un'altra ${ }^{116}$, e ciò benché la Description non abbia voluto porsi come testimonianza di una conquista militare fallita, ma come il suggello di un incontro pacifico, non conflittuale, tra due culture che si fondono armoniosamente entro una visione unitaria ${ }^{117}$. Centro e veicolo di questa imponente struttura ideologica sarà il futuro imperatore dei francesi, la cui gloria e prestigio troveranno ampia celebrazione nella Description, opera deputata alla "deificazione" della sua persona e del suo ruolo politico-militare, e il cui immenso successo investirà di luce riflessa l'intero progetto napoleonico. Numerosi, nel corso delle settemila pagine, sono i tributi offerti dai savants alla loro guida in terra d'Egitto:

Aussi le projet de ramener sur les rivages du Nil les sciences, si long-temps exilées, excita une reconnaissance universelle pour le Héros qui l'avoit conçu. Cette pensée portoit toute l'empreinte de son génie ${ }^{118}$.

Un grand homme répand sur tous ces objets l'éclat de sa gloire personnelle; il encourage par sa présence toutes les découvertes, ou plutôt il les suggère; et son esprit vaste s'applique en même temps, avec une incroyable facilité, à la guerre, à la politique, aux lois et aux sciences ${ }^{119}$.

(113) Ibid., pp. XXVIJ-XXIX.

(114) Cfr. EDward SAID, Orientalismo, l'immagine europea dell'Oriente, Milano, Feltrinelli, 2001, p. 97.

(115) Jean-Marie Carré, op. cit., introduzione, p. XXIX.

(116) Ibid., p. 44.

(117) SARga Moussa, La rencontre des Bédouins dans les "Observations sur la topographie de la presqu'île du Sinai" de Coutelle, in MARIE-NoËLle Bourguet (a cura di), op. cit., pp. 207-21.

(118) Description de l'Égypte, Préface et explication des planches, 1809, Préface Historique, p. XXXVII.

(119) Ibid., p. XLIII. 
I partecipanti all'impresa, pur ignari della loro destinazione e futuri compiti, erano ispirati tutti indistintamente da una fiducia unanime e la presenza di Napoleone o il suo solo nome bastavano ad alimentare in loro la speranza. Così ricorda Fourier:

Les Français qui devoient concourir à cette expédition avoient été rassemblés sur divers points des côtes de la Méditerranée: ils ignoroient le but vers lequel ils alloient être dirigés, et formoient à ce sujet les conjectures les plus opposées. L'ardeur guerrière, le feu de la jeunesse, l'incertitude, agitoient les cœurs; la présence d'un Héros inspiroit une confiance unanime. Son nom seul fixoit toutes les espérances; et l'on eut un exemple de cet ascendant suprême qu'exercent sur toutes les ames la gloire et le génie ${ }^{120}$.

Le vicende militari di cui fu teatro l'Egitto attirarono l'attenzione del mondo intero, la notizia si sparse rapidamente in Oriente e in Africa, oltre che in Europa:

Les traits multipliés de courage et de patience qui signalèrent ces campagnes, les dangers auxquels l'armée étoit sans cesse exposée, les fatigues inexprimables qu'elle endura, les talens supérieurs et le dévouement des généraux, excitèrent en France l'admiration et la reconnoissance publique ${ }^{121}$.

Non dimentichiamo, infine, che l'esplorazione scientifica e territoriale dell'Egitto da parte della compagine francese non è unicamente da leggersi nella prospettiva di una spedizione scientifica al servizio della conquista militare o di un abile pretesto per l'espansione territoriale. Il paziente e instancabile lavoro di scienziati e savants metterà infatti a disposizione delle generazioni future un prezioso e sistematico inventario, realizzato attraverso minuziose e metodiche classificazioni, rilevamenti e misurazioni per quanto atteneva la natura del territorio, il clima, la fauna, la flora, la produzione agricola e artigianale, il commercio, la distribuzione della popolazione. Così, a differenza degli studiosi del passato, che avevano trasformato la scienza antica in lezioni corrotte e dati imprecisi, gli studiosi francesi hanno realizzato uno studio rigorosamente scientifico, fondato su analisi critiche, obiettive e rigorose dei monumenti egizi, contenenti tutte le risposte ai quesiti più complessi ${ }^{122}$. Ciò che poi è stato inserito nell'opera è il risultato di comunicazioni reciproche tra i savants:

C'est une circonstance sur laquelle nous appelons particulièrement l'attention des lecteurs, parce qu'elle est très-remarquable. Aucun autre ouvrage, en effet, n'a présenté et ne présentera peut-être des dessins de monumens dont les plans, levés par des architectes et des ingénieurs, sont les résultats de cotes comparées, vérifiées et complétées réciproquement sur chacun d'eux. C'est pourquoi nous croyons devoir prévenir les voyageurs qui nous suivront, que ce seroit vainement qu'ils chercheroient à ajouter aux travaux publiés sur l'architecture dans la Description de l'Égypte ${ }^{123}$.

I suddetti elementi di studio e ricerca, unitamente all'investigazione archeologica delle vestigia faraoniche, hanno costituito per gli studiosi francesi un mezzo per la comprensione del loro presente e per la costruzione del futuro, attraverso un'opera di

(120) Ibid., p. XXXIX.

(121) Description de l'Égypte, Préface et explication des planches, 1809, Préface Historique, p. XL.

(122) Edme-FrançoIs Jomard, Mémoire sur le système métrique des anciens égyptiens, contenant des recherches sur leurs connoissances géométriques et sur les mesures des autres peuples de l'antiquité, in
Description de l'Égypte, Antiquités, Mémoires, vol. I, 1809, p. 778.

(123) Prosper Jollois, Édouard Devilliers, Description générale de Thèbes, in Description de l'Égypte, Antiquités, Descriptions, vol. I, 1809, nota 1, p. 207. 
modernizzazione ${ }^{124}$. Quasi sempre in condizioni precarie essi hanno annotato su fogli volanti o su propri blocchi da disegno quanto osservato, rilevando monumenti e siti e disegnandone dove possibile le planimetrie, senza che le difficoltà inficiassero la cura e correttezza dei risultati raggiunti: «Les difficultés que nous éprouvions, nous ont fait aisément sentir combien nous devions craindre de commettre de graves erreurs, et nous ont forcés à redoubler de soins et d'attention» ${ }^{125}$. Appellandosi alla propria volontà e determinazione e sfruttando le risorse vitali, personali e interiori per superare i numerosi ostacoli sul loro cammino, essi ci hanno per la prima volta restituito l'antico e moderno Egitto in tutto il suo splendore.

In conclusione, l'approccio della Description oscilla tra una soddisfatta esigenza di obiettività e polivalenza scientifica (che preveda uno scambio ed una interazione culturale pacifici) e una pretesa coscienza della superiorità tecnica, scientifica e ideologica dell'Occidente (che cela al suo interno istanze imperialiste). La complessità dell'esperienza da cui è scaturita quest'opera, unica nel suo genere e di cui molto altro ancora dovrebbe essere scritto, impone di considerare come riduttivo il tentativo di forzarne i confini entro i limiti di una categorizzazione o definizione troppo rigida, e dovrebbe invece spingere all'analisi attenta di tutte le sfumature che la colorano e i fecondi spunti successivamente sviluppati dalla cultura europea ottocentesca in ambito scientifico, artistico e letterario. Da essa prenderanno così l'avvio, grazie a tutta una serie di preziosi materiali che decifrati forniranno un bagaglio di nuove conoscenze, la scienza egittologica, e la riscoperta del gusto per l'arte egizia, tuttavia mai sopito, attraverso le imperanti tendenze dell'egittomania ed egittofilia. Ma questa è già un'altra storia.

BARBARA PELLEGRINELLI

(124) Annie Forgeau, Les repérages des sites de l'Égypte pharaoniques par les membres de la Commission des Sciences et des Arts, in MARIE-NoËLLE
BOURGUEt (a cura di), op. cit., pp. 33-52.

(125) Prosper Jollois, EDOUARD Devilliers, op. cit., p. 4. 